\title{
GREEN POTENTIAL OF EVANS TYPE ON ROYDEN'S COMPACTIFICATION OF A RIEMANN SURFACE
}

\author{
MITSURU NAKAI
}

\section{Introduction}

1. Let $R$ be a hyperbolic Riemann surface and $g^{w}(z)$ be the Green function on $R$ with its pole $w$ in $R$. We denote by $\mathscr{H}(R)$ the totality of sequences $\left(z_{n}\right)_{n=1}^{\infty}$ of points in $R$ not accumulating in $R$ and

$$
\lim \inf _{n \rightarrow \infty} g^{w}\left(z_{n}\right)>0 .
$$

Clearly the family $\mathscr{H}(R)$ is independent of the special choice of the pole $w$ and so $\mathscr{H}(R)$ is determined completely by the structure of $R$. We say that $R$ is regular (resp. irregular) if $\mathscr{F}(R)=\varnothing$ (resp. $\mathscr{F}(R) \neq \varnothing$ ). It is well recognized that for many problems, regular hyperbolic Riemann surfaces are more manageable than irregular ones. Hence it is important to provide tools to eliminate the irregularity in some sense. The main pourpose of this paper is to show the following ${ }^{1)}$

TheOREM 1. On any irregular hyperbolic Riemann surface $R$, there exists a positive harmonic function $u(z)$ satisfying the following three properties:

(1) $u(z)$ is an Evans function on $R$, i.e. $\lim _{n \rightarrow \infty} u\left(z_{n}\right)=\infty$ for any sequence $\left(z_{n}\right)_{n=1}^{\infty}$ belonging to the class $\mathscr{H}(R)$;

(2) $u(z)$ is quasi Dirichlet finite of the first order, i.e. there exists a finite positive constant $K$ such that $D_{R}(\min (u(z), c)) \leq K c$ for any poitive number $c$;

(3) $u(z)$ is singular, i.e. the greatest harmonic minorant of $\min (u(z), c)$ on $R$ is identically zero for any positive number $c$.

Here we make a remark to the property (1). Let $\left(R_{n}\right)_{n=1}^{\infty}$ be a normal exhaustion of $R$ and set $R_{0}=\varnothing$. For a positive number $a$ and a point $w$ in $R$ and a non-negative integer $n$, we set

Received December 25, 1963.

1) We published an outline of a part of this paper in [9]. We also published some results closely related to this paper in [8] and [10]. 


$$
V(w, a, n)=\left(z \in R ; g^{w}(z)>a\right)-\bar{R}_{n} \quad(n=0,1,2, \ldots) .
$$

Then the property (1) is equivalent to the following property:

(1') $\lim _{n \rightarrow \infty} \inf (u(z) ; z \in V(w, a, n))=\infty$ for an $y(w, a)$ such that $V(w, a, 0)$ is not a compact set in $R$.

In fact, let $\left(z_{n}\right)_{n=1}^{\infty}$ be in $\mathscr{F}(R)$. Then there exists a positive integer $n(m)$ such that $\left(z_{n}\right)_{n=n(m)}^{\infty}$ is contained in $V\left(w, 2^{-1} \lim _{\inf _{k \rightarrow \infty}} g^{w}\left(z_{k}\right), m\right)$ for any po. sitive integer $m$. From this, it follows that (1') implies (1). Convrsely, assume that (1) holds. If $\left(1^{\prime}\right)$ is not true for some $(w, a)$ with non-compact $V(w, a, 0)$, then there exists a sequence $\left(z_{n}\right)_{n=1}^{\infty}$ of points in $R$ such that there exists an increasing sequence $\left(k_{n}\right)_{n=1}^{\infty}$ of positive integers with $z_{n} \in V\left(w, a, k_{n}\right)$ and $\left(u\left(z_{n}\right)\right)_{n=1}^{\infty}$ is bounded. Clearly $\left(z_{n}\right)_{n=1}^{\infty}$ belongs to $\mathscr{H}(R)$ and so the boundedness of $\left(u\left(z_{n}\right)\right)_{n=1}^{\infty}$ contradicts (1).

2. Let $R^{\prime}$ be a parabolic Riemann surface and $\left(R_{n}^{\prime}\right)_{n=0}^{\infty}$ be a normal exhaustion of $R^{\prime}$ such that $R_{0}^{\prime}$ is a disc $(z ;|z|<1)$. Applying Theorem 1 to the hyperbolic Riemann surface $R=R^{\prime}-R_{0}^{\prime}$ which is clearly irregular, we get a positive harmonic function $u(z)$ on $R$ satisfying (1). Let $g^{w}(z)$ be Green's function on $R=R^{\prime}-\bar{R}_{0}^{\prime}$ with its pole $w$ in $R$. Then, since $R^{\prime}$ is parabolic, $\inf \left(g^{w}(z) ; z \in R^{\prime}-R_{1}^{\prime}\right)>0$. Hence from $\left(1^{\prime}\right)$, it follows that

$$
\operatorname{lin}_{n \rightarrow \infty} \inf \left(u(z) ; z \in R^{\prime}-R_{n}^{\prime}\right)=\infty \text {. }
$$

This is equivalent to that $u=\infty$ continuously at the Alexandroff point of $R^{\prime}$. Modifying $u$, it may be assumed that

$$
u(z)=0 \text { continuously at each point of } \partial R_{0}^{\prime},
$$

From this (3) follows, since $R^{\prime}$ is parabolic. We must remark that in the present case, without assuming (2), properties (1) and $\left(3^{\prime}\right)$ implies the more precise properties than (2). In fact, for any $c>0$, since $(z \in R ; u(z)<c)$ is closure compact in $R^{\prime}$ and $\int_{\partial R_{0^{\prime}}} * d u=\int_{u=c} * d u$, we get

$$
D_{R}(\min (u, c))=\int_{\partial R_{0^{\prime}} \cup(u=c)} u^{*} d u=\int_{u=c} c^{*} d u=\left(\int_{\partial R_{0^{\prime}}} * d u\right) c .
$$

Now by multiplying a suitable positive constant, we may assume $\int_{\partial R_{0^{\prime}}}{ }^{*} d u=2 \pi$. Let 


$$
s(z)= \begin{cases}u(z) & \text { on } R^{\prime}-R_{0}^{\prime} ; \\ -g_{0}^{w}(z) & \text { on } R_{0}^{\prime},\end{cases}
$$

where $g_{0}^{w}(z)$ is the Green function in $R_{0}^{\prime}$ with its pole $w$ in $R_{0}^{\prime}$. Then since

$$
\int_{\partial R_{0}+\partial\left(R-R_{0}\right)} * d s=0
$$

the equation $L(h-s)=h-s$, where $L$ is a normal linear operator of Sario [13]. has a solution on $R^{\prime}$ which is harmonic in $R-(w)$ and has the same singularity as $s(z)$ at $w$ and the Alexandroff point of $R^{\prime}$. Hence $h(z)$ has the negative logarithmic pole at $w$ and $h=\infty$ continuously at the Alexandorff point of $R^{\prime}$. This $h(z)$ is the so called Evans-Selberg's potential on $R^{\prime}$. Hence Theorem 1 contains generalized Evans-Selberg's theorem (see Evans [2], Selberg [14], Noshiro [12], Kuramochi [3], Nakai [8]).

3. Theorem 1 is a consequence of a more precise facts as mentioned below. Let $R$ be a hyperbolic Riemann surface and $R^{*}$ be its Royden's compactification. We denote by $\Gamma=R^{*}-R$, which is called Royden's boundary of $R$. We denote by $\Delta$ the totality of regular points in $\Gamma$ with respect to the Dirichlet problem, which is called (Royden's) harmonic boundary of $R$ and $\Delta \neq \varnothing$ if and only if $R$ is hyperbolic. It will be seen that the Green function $g^{w}(z)$ on $R$ can be extended to the Green kernel $g(p, q)$ on $R^{*}$ such that $g(z, p)$ is finitely continuous in $(z, p)$ of $R \times \Gamma$ and $g(z, w)=g^{w}(z)$ and as the function of $z, g(z, p)$ $\left(p \in R^{*}\right)$ is a non-negative singular harmonic function on $R-(p)$ and continuous on $R^{*}$. We set

$$
\Gamma_{0}=(p \in \Gamma ; g(z, p)>0 \text { on } R),
$$

which is an $F_{\sigma}$-set in $\Gamma$ and $\Gamma_{0} \neq \varnothing$ if and only if $R$ is irregular.

Theorem 2. Assume that $R$ is an irregular hyperbolic Riemann surface. Then there exists a unit positive regular Borel measure $\mu$ on $R^{*}$ satisfying the following six properties:

(4) there exists a sequence $\left(q_{n}\right)_{n=1}^{\infty}$ of points in $\Gamma_{0}$ such that $\mu\left(R^{*}-\left(q_{n}\right)_{n=1}^{\infty}\right)$ $=0$, i.e. $\mu=\sum_{i=1}^{\infty} t_{i} \varepsilon_{q_{i}}$, where $t_{i}>0$ and $\sum_{i=1}^{\infty} t_{i}=1$ and $\varepsilon_{q_{i}}$ is a unit point measure at $q_{i}$;

(5) $g_{\mu}(z)=\int g(z, q) d \mu(q)$ is a positive harmonic function on $R$;

(6) $D_{R}\left(\min \left(g_{\mu}(z), c\right)\right) \leq 2 \pi c$ for any positive number $c$; 
(7) $g_{\mu}(z)$ is continuous on $R^{*}$,

(8) $g_{\mu}(p)=\infty$ on $\Gamma_{0}$;

(9) $g_{\mu}(p)=0$ on $\Delta$.

4. In Chapter 1, we explain Royden's compactification and some of its fundamental properties. In Chapter 2, we define Green kernel on Royden's compactification and discuss its fundametal properties. In Chapter 3 , we treat transfinite diameters and modified Tychebycheff's constants for subsets of $R^{*}$ with respect to Green kernel. In Chapter 4, we complete the proofs of Theorems 1 and 2 .

5. Here we explain some notations and terminologies used in this paper. Functions (resp. continuous functions) on a space (resp. a topological space) considered in this paper are assumed to be mappings (resp. continuous mappings) of the space into the completed real line $[-\infty, \infty]$. For two numbers or functions $a$ and $b$, we denote

$$
a \cap b=\min (a, b) \text { and } a \cup b=\max (a, b) .
$$

Let $R$ be an open Riemann surface and $R^{*}$ be its Royden's compactification (see Section 1.1). For a subset $A$ in $R^{*}$, we denote by $\AA$ (resp. $\bar{A}$ ) the totality of inner point of $A$ (resp. the closure of $A$ ) considered in $R^{*}$. For a set $A$ in $R^{*}$, we denote by $\partial A$ the boundary of $A$ relative to $R$ (and not to $R^{*}$ ), i.e. $\partial A=(\bar{A}-A) \cap R$. Hence $\partial(A \cap R)=\partial A$. A normal exhaustion $\left(R_{n}\right)_{n=0}^{\infty}$ of $R$ is a sequence of closure compact subdomains $R_{n}$ of $R$ such that

$$
R_{n+1} \supset \bar{R}_{n} \text { and } R=\bigcup_{n=1}^{\infty} R_{n}
$$

and $R-\bar{R}_{n}(n=0,1,2, \ldots)$ have no component which is closure compact in $R$ and $R-\bar{R}_{0}$ is connected and each $\partial R_{n}$ consists of a finite number of mutually disjoint analytic closed Jordan curves. If $\partial R_{n}$ consists of a finite number of mutually disjont piece-wise analytic closed Jordan curves, then we say that $\left(R_{n}\right)_{n=1}^{\infty}$ is a normal exhaustion of $R$ with piece-wise analytic boundary. Finally, for two a.c.T functions $f$ and $g$ on an open set $G$ in $R$ (see Section 1.1), we set Dirichlet inner product and Dirichlet integral by

$$
D_{G}(f, g)=\iint_{G} d f \wedge^{*} d g \text { and } D_{G}(f)=\iint_{G} d f \wedge^{*} d f=\iint_{G}|\operatorname{grad} f(z)|^{2} d x d y,
$$

respectively, where $z=x+i y$ is a local parameter on $R$. If there is no afraid. 
of confusion, we simply write $D(f, g)$ and $D(f)$ instead of $D_{G}(f, g)$ and $D_{G}(f)$ respectively.

\section{Royden's compactification and some fundamental properties}

1.1. A real vlaued function $F(x, y)$ defined in $(a, b) \times(c, d)$ is said to be absolutely continuous in the sense of Tonelli (abreviated as a.c. T) if $F$ is continuous and if for any fixed $y$ in $(c, d)$ except a set of measure zero the function $x \rightarrow F(x, y)$ is absolutely continuous in the usual sense and the same is true if

$x$ and $y$ are interchanged and, further, $\frac{\partial}{\partial x} F(x, y)$ and $\frac{\partial}{\partial y} F(x, y)$ are integrable in any compact subset of $(a, b) \times(c, d)$. Since this notion is conformally invariant, this notion can be easily carried over Riemann surfaces using local parameters.

A (real) Royden's algebra $M(R)$ associated with a Riemann surface $R$ is the totality of real valued bounded continuous a.c.T functions $f$ on $R$ with finite Dirichlet integrals $D_{R}(f)$. This $M(R)$ is a Banach algebra with the usual algebraic operations and the norm $\|f\|=\sup _{R}|f|+\sqrt{D_{R}(f)}$ ([5]).

A sequence $\left(f_{n}\right)_{n=1}^{\infty}$ of functions on $R$ is said to converge to a function $f$ on $R$ in $C$ - (or $D$-) topology if $f_{n}$ converges to $f$ uniformly on each compact subset of $R$ (or $D_{R}\left(f_{n}-f\right) \rightarrow 0$ ), in notation $f=C-\lim _{n} f_{n} \quad\left(\right.$ or $f=D \cdot \lim _{n} f_{n}$ ). We also say that $\left(f_{n}\right)_{n=1}^{\infty}$ converges to $f$ in $B$ - (or $B D$-) topology if $\left(f_{n}\right)_{n=1}^{\infty}$ is bounded and converges to $f$ in $C$-topology (or $\left(f_{n}\right)_{n=1}^{\infty}$ converges to $f$ in $B$ - and $D$-topology), in notation $f=B \cdot \lim _{n} f_{n}$ (or $f=B D \cdot \lim _{n} f_{n}$ ). We remark that $M(R)$ is $B D$-complete ([5]).

We denote by $M_{0}(R)$ the totality of functions in $M(R)$ with compact support in $R$. We also denote by $M_{\Delta}(R)$ the $B D$-closure of $M_{0}(R)$ in $M(R)$. Clearly $M_{0}(R)$ and $M_{\Delta}(R)$ are ideals of $M(R)$. We also remark that $M_{\Delta}(R)$ is $B D$ complete.

The Royden's compactification $R^{*}$ of $R$ is a unique compact Hausdorff space containing $R$ as its open and dense subset and each function can be continuously extended to $R^{*}$ and $M(R)$ separates points in $R^{*}$. The set $\Gamma=R^{*}-R$ is called Royden's boundary of $R$ ([5], [1]). A part $\Delta$ of $R^{*}$ defined by

$$
\Delta=\left(p \in R^{*} ; f(p)=0 \text { for any } f \in M_{\Delta}(R)\right)
$$

is called the harmonic boundary of $R$. This set $\Delta$ is a compact subset of $\Gamma$ and $A \neq \varnothing$ if and only if $R$ is hyperbolic ([6]). This set $\Delta$ is the totality of regular points in $\Gamma$ with respect to Dirichlet problem ([7]). It holds the follow- 
ing duality $([6])$

$$
M_{\dot{\Delta}}(R)=(f \in M(R) ; f \text { vanishes on } \Delta) .
$$

1.2. For the application of Royden's compactification, the following four facts play the fundamental role. From the definition of a.c.T function, we have the following ([6], p. 69 in [1]):

Lemma 1.2.1. The algebra $M(R)$ forms a vector lattice with lattice operations $f \cup g$ and $f \cap g$.

Since $M(R)$ is a subalgebra of total algebra $B\left(R^{*}\right)$ of bounded continuous functions on $R^{*}$ with $M(R) \ni 1$ and $M(R)$ separates points in $R^{*}$, by Weierstrass-Stone's approximation theorem, we get:

Lemma 1.2.2. The algebra $M(R)$ is dense in $B\left(R^{*}\right)$ with respect to the uniform convergence topology on $R^{*}$.

Lemma 1.2.3 (Kusunoki-Mori [5]). Let $U$ be a subdomain of $R$ sunch that $\bar{U} \cap \Delta=\varnothing$ and $\partial U$ consists of at most countable number of mutually disjoint analytic Jordan curves not ending and not accumulating in $R$. Then the double $\hat{U}$ of $U$ along $\partial U$ is a compact or parabolic Riemann surface.

LeMmA 1.2.4 (Nakai [6]). Suppose that $u(z)$ is a harmonic function bounded from below (or above) defined on a subdomain $U$ of $R$ such that $\partial U$ (which may be empty) consists of at most a countable number of Jordan curves not accumulating in $R$. If $u$ satisfies

$$
\lim \inf _{U \ni z \rightarrow \zeta} u(z) \geq m\left(\text { or } \lim \sup _{U \ni z \rightarrow \zeta} u(z) \leq M\right)
$$

at any point $\zeta$ in $\partial U \cup(\bar{U} \cap \Delta)$, then $u \geq m$ (or $u \leq M)$ on $U$.

1.3. Since $R$ is dense in $R^{*}$, we may say that a function $f$ defined on $R$ is continuous on $R^{*}$ if $f$ is continuously extended to $R^{*}$. We say that a nonnegative function $f$ on $R$ is quasi Dirichlet finite on $R$ if $f \cap c$ is a.c.T and $D_{R}(f \cap c)<\infty$ for any positive number $c$. It is easy to see that $\alpha f_{1}+\beta f_{2}$ $(\alpha, \beta>0)$ is quasi Dirichlet finite on $R$ along with $f_{1}$ and $f_{2}$.

Lемма 1.3.1. If $f$ is quasi Dirichlet finite continuous function on. $R$, then $f$ is continuous on $R^{*}$.

Proof. For any $n=1,2, \ldots, f \cap n$ can be continuously extended to $R^{*}$. We denote by $f_{n}$ the extended function. Let $h(p)=\lim _{n \rightarrow \infty} f_{n}(p)$, whose ex. 
istence is clear, since $\left(f_{n}\right)_{n=1}^{\infty}$ is non-decreasing on $R^{*}$. Assume that $h\left(p_{0}\right)<\infty$ $\left(p_{0} \in R^{*}\right)$. Then there exists an $n$ such that $h\left(p_{0}\right)<n$. Since $f_{n}$ is continuous at $p_{0}$, there exists a neighborhood $U$ of $p_{0}$ in $R^{*}$ such that $f_{n}(p)<n(p \in U)$. If $m>n$, then $(f \cap m) \cap n=f \cap n$ on $R$. Hence $f_{m} \cap n=f_{n}$ on $R^{*}$. Thus $f_{m}(p) \cap n=f_{n}(p)<n(p \in U)$ implies that $f_{m}(p)=f_{n}(p)$ in $U$ and so by making $m \nearrow \infty$, we get $h(p)=f_{n}(p)$ in $U$. Thus $h$ is continuous at $p_{0}$. Next suppose that $h\left(p_{0}\right)=\infty$. Then for any $c>0$, there exists an $f_{n}$ such that $f_{n}\left(p_{0}\right)>c$. Since $f_{n}$ is continuous on $R^{*}$, there exists a neighborhood $V$ of $p_{0}$ such that $f_{n}(p)>c$ on $V$. As $h \geq f_{n}$ on $R^{*}$, so $h(p)>c(p \in V)$. This shows that $h$ is continuous at $p_{0}$. Hence $h$ is continuous on $R^{*}$. Clearly $h(z)=\lim _{n \rightarrow \infty} f_{n}(z)=$ $\lim _{n \rightarrow \infty} f(z) \cap n=f(z)$ on $R$. Thus $h$ is a continuous extention of $f$. In other words, $f$ is continuous on $R^{*}$.

1.4. We prove three more lemmas which plays an important role in our paper.

LeMMA 1.4.1. Let $\left(\varphi_{n}\right)_{n=1}$ be a sequence in $M_{\Delta}(R)$ such that $\varphi=B-\lim _{n} \varphi_{n}$ on $R$ and $\varphi$ is a.c.T on $R$ and $\lim _{n} D_{K}\left(\varphi_{n}-\varphi\right)=0$ for each compact subset $K$ of $R$ and $D_{R}\left(\varphi_{n}\right) \leq A<\infty(n=1,2, \ldots)$. Then $\varphi$ belongs to $M_{\Delta}(R)$ and $D_{R}(\varphi, f)$ $=\lim _{n} D\left(\varphi_{n}, f\right)$ for each $f$ in $M(R)^{2}$.

Proof. Let $\left(R_{n}\right)_{n=0}^{\infty}$ be a normal exhaustion of $R$. We take a continuous function $\phi_{n}$ on $R$ such that

$$
\phi_{n}= \begin{cases}\varphi_{n} & \text { on } R_{0} ; \\ \text { harmonic } & \text { in } R_{1}-R_{0} \\ 0 & \text { on } R-R_{1},\end{cases}
$$

$n=1,2, \ldots, \infty$, where we set $\varphi_{\infty}=\varphi$. Then clearly $\phi_{n} \in M_{0}(R) \subset M_{\Delta}(R)$. Let $\varphi_{n}^{\prime}=\varphi_{n}-\phi_{n}$ and $\varphi^{\prime}=\varphi-\phi_{\infty}$. Then $\varphi_{n}^{\prime}$ and $\varphi^{\prime}$ satisfy the assumption of Lemma 1.4.1. If the conclusion of Lemma 1.4 .1 is valid for $\varphi_{n}^{\prime}$ and $\varphi^{\prime}$, then the same is true for $\varphi_{n}$ and $\varphi$. Hence to prove our lemma, we may assume without loss of generality that $\varphi=\varphi_{n}=0$ on $R_{0}(n=1,2, \ldots)$.

Let $\Gamma^{2}(R)$ be the real Hilbert space of all real first order measurable differentials $\alpha$ such that $\|\alpha\|^{2}=\iint_{R} \alpha \wedge^{*} \alpha<\infty$. We denote $(\alpha, \beta)=\iint_{R} \alpha \wedge^{*} \beta$. Notice

2) Mr. M. Kawamura proved that this Lemma is true without assuming that $\varphi$ is a.c. $\mathrm{T}$ and $\lim _{n} D_{K}\left(\varphi_{n}-\varphi\right)=0$. 
that $d f \in \Gamma^{2}(R)$ if $f \in M(R)$. Then $\left(d \varphi_{n}\right)_{n=1}^{\infty} \subset \Gamma^{2}(R)$ and $\left\|d \varphi_{n}\right\|^{2} \leq A \quad(n=1,2$, ...). Hence by the weak compactness of bounded set in the Hilbert space, any subsequence of $\left(d \varphi_{n}\right)_{n=1}^{\infty}$ possesses a waekly convergent subsequence. We show that the weak limit of any weakly convergent subsequence is $d \varphi$. If this can be shown, then we can conclude that $\left(d \varphi_{n}\right)_{n=1}^{\infty}$ itself converges weakly to $d \varphi$.

Assume that $\left(d \varphi_{n_{k}}\right)_{k=1}^{\infty}$ is wearkly convergent. Then $\beta \rightarrow \lim _{k}\left(\beta, d \varphi_{n_{k}}\right)$ is a bounded linear functional on $\Gamma^{2}(R)$. By the reflexivity of the Hilbert space, there exist an $\alpha$ in $\Gamma^{2}(R)$ such that for any $\beta \in \Gamma^{2}(R),(\beta, \alpha)=\lim _{k}\left(\beta, d \varphi_{n_{k}}\right)$. Assume that $\beta=0$ outside $R_{m}$. Then

$$
\left|\left(\beta, d \varphi-d \varphi_{n_{k}}\right)\right| \leq\|\beta\| \cdot\left\|d \varphi-d \varphi_{n_{k}}\right\|_{R_{m}}=\|\beta\| \sqrt{D_{R_{m}}\left(\varphi-\varphi_{n_{k}}\right)} \rightarrow 0
$$

as $k \nearrow \infty$. Thus $(\beta, d \varphi)=\lim _{k}\left(\beta, d \varphi_{n_{k}}\right)$. Hence $(\beta, d \varphi-\alpha)=0$ for any $\beta \in \Gamma^{2}(R)$ with $\beta=0$ outside $R_{m}$. This shows that $\alpha=d \varphi$ on $R_{m}$. Since $R_{m}$ is arbitrary, $\alpha=d \varphi$ on $R$ and $\lim _{k} d \varphi_{n_{k}}=d \varphi$ (weakly).

Hence we obtain that $\lim _{n} d \varphi_{n}=d \varphi$ (weakly) and in particular, $\lim _{n} D_{R}\left(\varphi_{n}, f\right.$ ) $=D_{R}(\varphi, f)$ for any $f$ in $M(R)$ and $D_{R}(\varphi)<\infty$. As $\varphi=B$ - $\lim \varphi_{n}$, so $\varphi$ is continuous on $R$ and so $\varphi \in M(R)$.

Finally we prove that $\varphi \in M_{\Delta}(R)$. Let $u_{m}$ be continuous on $R$ such that

$$
u_{m}= \begin{cases}\varphi=0 & \text { on } R_{0} \\ \text { harmonic } & \text { in } R_{m}-R_{0} \\ \varphi & \text { on } R-R_{m} .\end{cases}
$$

As we have

$$
D_{R}\left(u_{m+p}-u_{m}, u_{m+p}\right)=\int_{\partial R_{0} \cup \partial R_{m+p}}\left(u_{m+p}-u_{m}\right)^{*} d u_{m+p}=0
$$

so $D_{R}\left(u_{m+p}-u_{m}\right)=D_{R}\left(u_{m}\right)-D_{R}\left(u_{m+p}\right)$. Hence $\left(u_{m}\right)_{m=1}^{\infty}$ is $D$-convergent. Since $u_{m}=0$ on $R_{0},\left(u_{m}\right)_{m=1}^{\infty}$ converges to a function $u$ in $C$-topology, where $u=0$ on $R_{0}$ and harmonic in $R-R_{0}$. Moreover, since $\left|u_{m}\right| \leq \sup _{R}|\varphi|, \quad u=B-\lim _{m} u_{m}$ on $R$. Hence

$$
u=B D \cdot \lim _{m} u_{m} \quad \text { on } R \text {. }
$$

Let $\phi_{m}=\varphi-u_{m}$ and $\phi=\varphi-u$. Then

$$
\phi=B D \cdot \lim _{m} \phi_{m}
$$

and as $\phi_{m} \in M_{0}(R)$, so $\phi \in M_{\Delta}(R)$. Since 


$$
D_{R}\left(u_{m}, \phi_{m}\right)=\int_{\partial R_{0} \cup \partial R_{m}} \phi_{m}^{*} d u_{m}=0,
$$

$D_{R}(u, \phi)=0$. Next, since $\varphi_{n} \in M_{\Delta}(R)$, we can find $\varphi_{n, k} \in M_{0}(R)$ such that $\varphi_{n}=B D$ - $\lim _{k} \varphi_{n, k}$. Suppose that the support of $\varphi_{n, k}$ is contained in $R_{n}(k)$. Then

$$
\left|D\left(u, \varphi_{n, k}\right)\right|=\left|\int_{\partial R_{0} \cup \partial R_{n}(k)} \varphi_{n, k} * d u\right| \leq \sup _{\partial R_{0}}\left|\varphi_{n, k}\right| \cdot \int_{\partial R_{0}} * d u \rightarrow 0
$$

as $k \nearrow \infty$. Hence $D\left(u, \varphi_{n}\right)=0(n=1,2, \ldots)$. Thus $D(u, \varphi)=\lim _{n} D\left(u, \varphi_{n}\right)=$ 0 . Hence from

$$
D(\varphi, u)=D(u, u)+D(\phi, u),
$$

we conclude that $D(u)=0$. As $u=0$ on $R_{0}$, so $u \equiv 0$ on $R$ and so $\varphi=\phi \in M_{\Delta}(R)$.

Q.E.D.

Lemma 1.4.2 [Generalized Dirichlet principle]. Let $K$ be a compact set in $R^{*}$ (which may be empty) such that $K \cap \Delta=\varnothing$ and $\overline{K \cap R}=K$ and $\partial K$ consists of at most a countable number of piece-wise analytic curves not accumulating in $R$. Assume that $f \in M(R)$. Then there exists a unique $u$ in $M(R)$ such that

$$
u= \begin{cases}f & \text { on } \Delta \cup K \\ \text { harmonic } & \text { in } R-K\end{cases}
$$

and $D_{R}(f)=D_{R}(u)+D_{R}(f-u)$ and

$$
D_{R}(u)=\min \left(D_{R}(h) ; h \in M(R) \text { and } h=f \text { on } \Delta \cup K\right) .
$$

Proof. Let $\left(R_{n}\right)_{n=1}^{\infty}$ be a normal exhaustion of $R$ and $u_{n}$ be continuous on $R$ such that

$$
u_{n}= \begin{cases}f & \text { on } R-\left(R_{n}-K\right) ; \\ \text { harmonic } & \text { in } R_{n}-K .\end{cases}
$$

Clearly $u_{n} \in M(R)$ and $\left|u_{n}\right| \leq \sup _{R}|f|$ on $R(n=1,2, \ldots)$. Hence by choosing a suitable subsequence, we may assume that $\left(u_{n}\right)_{n=1}^{\infty}$ is $B$-convergent on $R$. We set $u=B-\lim _{n} u_{n}$ on $R$. Then $u=f$ on $K \cap R$ and harmonic in $R-K$. Clearly $f-u_{n} \in M_{0}(R)$ and $f-u_{n}=0$ on $R-\left(R_{n}-K\right)$. Hence

$$
D_{R}\left(f-u_{n}, u_{n}\right)=\int_{\partial\left(R_{n}-K\right)}\left(f-u_{n}\right)^{*} d u_{n}=0
$$

and so $D_{R}(f)=D_{R}\left(u_{n}\right)+D_{R}\left(f-u_{n}\right)$ and in particular, $D_{R}\left(u_{n}\right) \leq D_{R}(f)$. Similarly, 
$D_{R}\left(u_{n}-u_{n+p}, u_{n+p}\right)=0$ and so $D_{R}\left(u_{n}-u_{n+p}\right)=D_{R}\left(u_{n}\right)-D_{R}\left(u_{n+p}\right)$. Thus $\left(u_{n}\right)_{n=1}^{\infty}$ is $D$-convergent and so $u=D \cdot \lim _{n} u_{n}$ on $R$. Hence

$$
u=B D \cdot \lim _{n} u_{n} \quad \text { on } R \text {. }
$$

Thus $u \in M(R)$ and $u$ is continuous on $R^{*}$. From this, $u=f$ on $K \cap R$ implies $u=f$ on $K=\overline{K \cap R}$. Moreover, $f-u=B D \cdot \lim _{n}\left(f-u_{n}\right)$ and $f-u_{n} \in M_{0}(R)$ implies that $f-u \in M_{\Delta}(R)$ or $u=f$ on $\Delta$. From $D_{R}(f)=D_{R}\left(u_{n}\right)+D_{R}\left(f-u_{n}\right)$, we get $D_{R}(f)=D_{R}(u)+D_{R}(f-u)$ and $D_{R}(u) \leq D_{R}(f)$. The unicity of such a $u$ follows from Lemma 1.2.4.

Next let $h \in M(R)$ with $h=f$ on $\Delta \cup K$. Construct $v \in M(R)$ for $h$ such that $v=h$ on $\Delta \cup K$ and harmonic in $R-K$. Then $D_{R}(v) \leq D_{R}(h)$. As $v=h=$ $f=u$ on $\Delta \cup K$, the unicity assures that $v=u$ on $R$. Thus $D_{R}(u) \leq D_{R}(h)$.

Q.E.D.

Lemma 1.4.3. Let $K$ and $K^{\prime}$ be compact sets in $R^{*}$ such that $\stackrel{\circ}{K}^{\prime} \supset K$ and $K^{\prime} \cap \Delta=\phi$ and $\overline{K \cap R}=K$ and $\overline{K^{\prime} \cap R}=K^{\prime}$ and relative boundaries $\partial K$ and $\partial K^{\prime}$ consist of at most a counsable number of disjoint analytic Jordan curves not ending and not accumulating in $R$. Then

(1.4.1) there exists a unique $u$ in $M(R)$ such that $u=1$ on $K$ and $u=0$ on $\triangle$ and $u$ is harmonic in $R-K$;

(1.4.2) $D_{R}(u)=\int_{\partial K}^{*} d u$;

(1.4.3) if $\int_{\partial K^{\prime}}\left|{ }^{*} d u\right|<\infty$, then $\int_{\partial K^{\prime}} * d u=\int_{\partial K}{ }^{*} d u$.

Proof. Let $\left(R_{n}\right)_{n=0}^{\infty}$ be a normal exhaustion of $R$ and $F$ be a compact set in $R^{*}$ such that $\stackrel{F}{\triangle} \triangle$ and $\overline{F \cap R}=F$ and $F \cap K=\varnothing$ and $\partial F$ consists of at most a countable number of piece-wise analytic Jordan curves not ending and not accumulating in $R$. We put $F_{k}=F-R_{k}$. By Lemmas 1.2 .1 and 1.2.2, we can find a function $f$ in $M(R)$ such that $f=0$ on $F$ and $f=1$ on $K$. By Lemma 1.4.2, there exists a function $\boldsymbol{u}_{k}$ in $M(R)$ such that $\boldsymbol{u}_{k}=f$ on $K \cup \overline{\partial F}_{k} \cup \Delta$ and $u$ is harmonic in $R-K \cup \partial F_{k}$. By Lemma 1.2.4, $u_{k}=0$ on $\stackrel{\circ}{F}_{k} \cap R$. Hence

$$
\boldsymbol{u}_{k}= \begin{cases}1 & \text { on } K \\ \text { harmonic } & \text { in } R-K \cup F_{k} \\ 0 & \text { on } F_{k}\end{cases}
$$

and by Lemma 1.4.2, 


$$
D_{R}\left(u_{k}\right) \leq D(f) .
$$

Next let $u_{k, n}$ be continuous on $\overline{R_{n}-K-F_{k}}(n>k)$ and harmonic in $R_{n}-K$ $-F_{k}$ such that $u_{k, n}=1$ on $\partial K \cap R_{n}$ and $u_{k, n}=0$ on $\partial F_{k} \cap R_{n}$ and $\frac{\partial}{\partial \nu} u_{k, n}=0$ on $\partial R_{n}-K-F_{k}$. We set $u_{k, n}=0$ on $F_{k}$ and $u_{k, n}=1$ on $K$. Then, since

$$
D_{R_{n}}\left(u_{k, n+p}-u_{k, n}, u_{k, n}\right)=\int_{\partial\left(R_{n}-K-F_{k}\right)}\left(u_{k, n+p}-u_{k, n}\right)^{*} d u_{k, n}=0,
$$

we get

$$
D_{R_{n}}\left(u_{k, n+p}-u_{k, n}\right)=D_{R_{l}}\left(u_{k, n+p}\right)-D_{R_{n}}\left(u_{k, n}\right) \leq D_{R_{n+p}}\left(u_{k, n+p}\right)-D_{R_{n}}\left(u_{k, n}\right) .
$$

On the other hand,

$$
D_{R_{n}}\left(u_{k}-u_{k, n}, u_{k, n}\right)=\int_{\partial\left(R_{n}-K-F_{k}\right)}\left(u_{k}-u_{k, n}\right)^{*} d u_{k, n}=0
$$

implies that $D_{R_{n}}\left(u_{k, n}\right)=D_{R_{n}}\left(u_{k}, u_{k, n}\right) \leq \sqrt{D_{R_{n}}\left(u_{k}\right)} \cdot \sqrt{D_{R_{n}}\left(u_{k, n}\right)}$, or

$$
D_{R_{n}}\left(u_{k, n}\right) \leq D_{R_{n}}\left(u_{k}\right) \leq D_{R}(f) .
$$

As $\left(D_{R_{n}}\left(u_{k, n}\right)\right)_{n>k}$ is non-decreasing and bounded, so

$$
\lim _{n} D_{R_{n}}\left(u_{k, n+p}-u_{k, n}\right)=0 .
$$

Since $\left(u_{k, n}\right)_{n>k}$ is bounded and $u_{k, n}$ is harmonic in $R_{n}-K-F_{k}$ and 0 on $\partial F_{k}$ and 1 on $\partial K, \lim _{n} u_{k, n}$ is a harmonic function in $R-K-F_{k}$ which equals 0 on $\partial F_{k}$ and 1 on $\partial K$. Hence by Lemma 1.2.4, $\boldsymbol{u}_{k}=\lim _{n} \boldsymbol{u}_{k, n}$ in $R-K-F_{k}$ and so on $R$. Hence by Fatou's Lemma,

$$
D_{R_{n}}\left(\boldsymbol{u}_{k}-\boldsymbol{u}_{k, n}\right) \leq \lim \inf _{p} D_{R_{n}}\left(u_{k, p}-u_{k, n}\right)
$$

and so

$$
\lim _{n} D_{R_{n}}\left(u_{k}-u_{k, n}\right) \leq \lim _{n}\left(\lim \inf _{p} D_{R_{n}}\left(u_{k, p}-u_{k, n}\right)\right)=0
$$

and also

$$
\lim _{n} D_{R_{n}}\left(u_{k, n}\right)=D_{R}\left(u_{k}\right) .
$$

Similarly as before, since

$$
D_{R_{n}}\left(u_{k+q, n}-u_{k, n}, u_{k+q, n}\right)=\int_{\partial\left(R_{n}-F_{k+q}-K\right)}\left(u_{k+q, n}-u_{k, n}\right)^{*} d u_{k+q, n}=0 \quad(n>k+q),
$$

we have

$$
D_{R_{m}}\left(u_{k+q, n}-u_{k, n}\right) \leq D_{R_{n}}\left(u_{k+q, n}-u_{k, n}\right)=D_{R_{n}}\left(u_{k, n}\right)-D_{R_{n}}\left(u_{k+q, n}\right) \quad(n>m)
$$


and so by making $n \nearrow \infty$ and then $m \nearrow \infty$, we get

$$
D_{R}\left(u_{k+q}-u_{k}\right) \leq D_{R}\left(u_{k}\right)-D_{R}\left(u_{k+q}\right) .
$$

As $D_{R}\left(u_{k}\right)=\lim _{n} D_{R_{n}}\left(u_{k, n}\right) \leq D_{R}(f)$, so

$$
\lim _{k} D_{R}\left(\boldsymbol{u}_{k+q}-u_{k}\right)=0 .
$$

Since $u_{k}=1$ on $K$ and $\left(u_{k}\right)_{k=1}^{\infty}$ is bounded and $u_{k}$ is harmonic on $R-K-F_{k}$, $\left(u_{k}\right)_{k=1}^{\infty}$ converges to a function $u$ on $R$ in $B$-topology and $u$ is harmonic on $R-K$ and $u=1$ on $K$. Moreover

$$
u=B D \cdot \lim _{k} u_{k}
$$

and $u_{k} \in M_{\Delta}(R)$ implies $u=0$ on $\Delta$. This $u$ is the desired in (1.4.1) and the unicity follows from Lemma 1.2.4.

Next we prove (1.4.2). From (1.4.1), we can find a function $e$ in $M_{\mathbf{A}}(R)$ such that

$$
e= \begin{cases}1 & \text { on } \overline{R-F}_{k} \\ \text { harmonic } & \text { in } \stackrel{\circ}{F}_{k} \\ 0 & \text { on } \Delta .\end{cases}
$$

Clearly $e$ is superharmonic on $R$. Let $h_{m}$ be continuous on $R$ such that

$$
h_{m}= \begin{cases}e & \text { on } R-R_{m} \\ \text { harmonic } & \text { in } R_{m}\end{cases}
$$

Then it is easily seen that $0 \leq h_{m+p} \leq h_{m} \leq e$ on $R$ and since

$$
D_{R}\left(h_{m}-h_{m+p}, h_{m+p}\right)=\int_{\partial R_{m+p}}\left(h_{n}-h_{m+p}\right) * d h_{m+p}=0,
$$

we get $D_{R}\left(h_{m}-h_{m+p}\right)=D_{R}\left(h_{m}\right)-D_{R}\left(h_{m+p}\right)$. Hence if we put $h=\lim _{m} h_{m}$ on $R$, then $h=B D-\lim _{m} h_{m}$ on $R$ and so $h \in H B D(R) \subset M(R)$ and as $0 \leq h \leq e$, so $h=0$ on 4 . Hence by Lemma $1.2 .4, h \equiv 0$. Hence if we put

$$
\varphi_{m}=e-h_{m},
$$

then $0 \leq \varphi_{m} \leq \varphi_{m+p} \leq e \leq 1$ on $R$ and $e=B D-\lim _{m} \varphi_{m}$ and the support of $\varphi_{m}$ is contained in $\bar{R}_{m}$. Now we have

$$
\begin{aligned}
D_{R}\left(u_{k} \varphi_{m}, u_{k}\right) & =\lim _{n} D_{R_{n}}\left(u_{k, n} \varphi_{m}, u_{k, n}\right) \\
& =\lim _{n} \int_{\partial\left(R_{n}-K-F_{k}\right)} u_{k, n} \varphi_{m}{ }^{*} d u_{k, n}
\end{aligned}
$$




$$
=\lim _{n} \int_{\partial K \cap R_{m}} \varphi_{m}^{*} d u_{k, n}
$$

As $u_{k, n} \rightarrow u_{k}(n \rightarrow \infty)$ uniformly on $R_{m}$, so ${ }^{*} d u_{k, n} \rightarrow^{*} d u_{k}$ on $\partial K \cap R_{m}$ uniformly and so

$$
D\left(u_{k} \varphi_{m}, u_{k}\right)=\int_{\partial K \cap R_{m}} \varphi_{m}^{*} d u_{k}
$$

We can easily show that

$$
u_{k}=e u_{k}=B D \cdot \lim _{m} \varphi_{m} u_{k}
$$

on $R$. Since ${ }^{*} d u_{k}>0$ on $\partial K$ and $0 \leq \varphi_{m} \leq 1$, we get $\varphi_{m}{ }^{*} d u_{k} \leq{ }^{*} d u_{k}$ on $\partial K$. Hence $D\left(u_{k} \varphi_{m}, u_{k}\right) \leq \int_{\partial K} * d u_{k}$ and so

$$
D\left(u_{k}\right) \leq \int_{\partial K}^{*} d u_{k}
$$

On the other hand, by Fatou's lemma,

$$
\int_{\partial K} * d u_{k} \leq \lim \inf _{m} \int_{\partial K \cap R_{m}} \varphi_{m}^{*} d u_{k}=\lim _{m} D\left(u_{k} \varphi_{m}, u_{k}\right)=D\left(u_{k}\right)
$$

This shows that $D\left(u_{k}\right)=\int_{\partial K}{ }^{*} d u_{k}$. By Lemma 1.2.4, $u_{k} \leq u_{k+p}$ on $R$ and as $\boldsymbol{u}_{k}=\boldsymbol{u}_{k+p}=1$ on $\partial K$, so ${ }^{*} d u_{k} \geq{ }^{*} d \boldsymbol{u}_{k+p} \geq 0$ on $\partial K$. Hence ${ }^{*} d u_{k} \rightarrow^{*} d u$ and by Lebesgue's convergence theorem,

$$
\int_{\partial \boldsymbol{K}}^{*} d u=\lim _{k} \int_{\partial K}^{*} d u_{k}=\lim _{k} D\left(u_{k}\right)=D(u),
$$

which proves (1.4.2).

Finally we prove (1.4.3). Let $R \cap\left(\stackrel{\circ}{K}^{\prime}-K\right)=\bigcup_{n} S_{n}$ be the decomposition into connected components. If we have $\int_{\bar{s}_{n} \cap \partial K} * d u=\int_{\bar{s}_{n \cap \partial K^{\prime}}} * d u$, then since $\int_{\partial K}|* d u|$ and $\int_{\partial K}|* d u|$ are finite, we get

$$
\int_{\partial K}^{*} d u=\sum_{n} \int_{\bar{s}_{n \cap \partial K}} * d u=\sum_{n} \int_{S_{n} \cap \partial K^{\prime}} * d u=\int_{\partial K^{\prime}} * d u
$$

Hence we may assume without loss of generality that $\left(\stackrel{\circ}{K}^{\prime}-K\right) \cap R$ is a domain. Let $T$ be the double of $\left(\hat{K}^{\prime}-K\right) \cap R$ with respect to $\partial K$ and $\partial K^{\prime}$ and $\left(T_{n}\right)_{n=1}^{\infty}$ be a normal exhaustion of $T$ and $T_{0}$ is a disc in $\left(\stackrel{\circ}{K}^{\prime}-K\right) \subset R$ such that $\bar{T}_{0} \subset$ $\left(\hat{K}^{\prime}-K\right) \cap R \cap T_{1}$. For convinience, we set $\gamma_{n}=\partial T_{n} \cap\left(K^{\prime}-\stackrel{K}{K}\right) \cap R$. We take 
a continuous function $v_{n}$ on $T$ such that

$$
v_{n}= \begin{cases}1 & \text { on } T_{0} \\ \text { harmonic } & \text { in } T_{n}-T_{0} \\ 0 & \text { on } T-T_{n} .\end{cases}
$$

Since $\overline{\left(\dot{K}^{\prime}-K\right) \cap R} \cap \Delta=\phi, T$ is parabolic by Lemma 1.2 .3 and so

$$
B D-\lim _{n} v_{n}=1
$$

on $T$. Since ${ }^{*} d v_{n}=0$ on $\partial K \cup \partial K^{\prime}$ and $\int_{r_{0}}{ }^{*} d u=0$, we get

$$
D_{(\stackrel{\mathrm{K}}{\mathrm{K}} \cap \cap R-K)}\left(v_{n}, u\right)=\int_{\left(\partial K-\partial K^{\prime}\right) \cup \gamma_{0} \cup \gamma_{n}} v_{n}^{*} d u=\int_{\partial \mathrm{K}-\partial K^{\prime}} v_{n}^{*} d u .
$$

As $v_{n}{ }^{*} d u \rightarrow \rightarrow^{*} d u$ and $\left|v_{n}{ }^{*} d u\right| \leq^{*} d u$ on $\partial K \cap \partial K^{\prime}$ and $\left|{ }^{*} d u\right|$ is integrable on $\partial K \cup \partial K^{\prime}$, so by Lebesgue's convergence theorem,

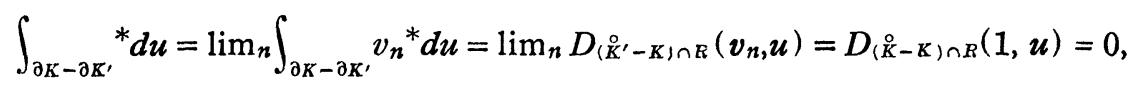

which shows $\int_{\partial K}{ }^{*} d u=\int_{\partial K^{\prime}} * d u$, i.e. (1.4.3). This completes the proof.

\section{Green kernel on Royden's compactification}

2. 1. Let $R$ be a hyperbolic Riemann surface and $g^{w}(z)$ be the Green function on $R$ with its pole $w$ in $R$. Let $\left(R_{n}\right)_{n=0}^{\infty}$ be a normal exhaustion of $R$ with $w$ in $R_{0}$ and $g_{n}^{w}(z)$ be the Green function on $R_{n}$. We set $g_{n}^{w}(z)=0$ for $z$ in $R-R_{n}$. By definition, $g_{n}^{w}(z) \nearrow g^{w}(z)$ on $R$ and $u_{n}(z)=g^{w}(z)-g_{n}^{w}(z)$ is bounded and $B-\lim _{n} u_{n}(z)=0$ on $R$. Since $u_{n}$ is harmonic in $R_{n}$ and $u_{n}=g^{w}$ on $R-R_{n}$,

$$
D_{R}\left(u_{n}-u_{n+p}, u_{n+p}\right)=\int_{\partial R_{n+p}}\left(u_{n}-u_{n+p}\right)^{*} d u_{n+p}=0 .
$$

Hence $D_{R}\left(u_{n}-u_{n+p}\right)=D_{R}\left(u_{n}\right)-D_{R}\left(u_{n+p}\right)$. From these, we conclude that $B D$. $\lim _{n} u_{n}=0$. In other words, $g_{n}^{w}(z) \nearrow g^{w}(z)$ on $R$ and $D_{R}\left(g_{n}^{w}(z)-g^{w}(z)\right) \rightarrow 0$ as $n \nearrow \infty$. Hence

$$
g^{w}(z) \cap c=B D \cdot \lim _{n} g_{n}^{w}(z) \cap c
$$

on $R$ for any $c>0$ (cf. p. 78, Satz 7.4 in [1]). As $g_{n}^{w}(z) \cap c \in M_{0}(R)$ and $D_{R}\left(g_{n}^{w}(z) \cap c\right)=2 \pi c$, so we get

$$
g^{w}(z) \cap c \in M_{\Delta}(R) \quad \text { and } \quad D_{R}\left(g^{w}(z) \cap c\right)=2 \pi c
$$


for any $c>0$. Hence $g^{w}(z)$ is quasi Dirichlet finite and so by Lemma 1.3.1, $g^{w}$ is continuous on $R^{*}$. As $g^{w}(p) \cap c=0$ on $\Delta$ for any $c>0$, so $g^{w}(p)=0$ on $\Delta$. Since $g^{w}(\dot{z})=g^{z}(w)$ on $R \times R$, we may define $g^{p}(z)$ as $g^{z}(p)$ for $p$ in $\Gamma$. We set

$$
\Gamma_{0}=\left(p \in \Gamma ; g^{w}(p)>0\right)
$$

for a fixed $w$ in $R$. By Harnack's inequality, we can easily see that $\Gamma_{0}$ is independent of the special choice of $w$ in $R$. Hence $\Gamma_{0}=\left(p \in \Gamma ; g^{w}(p)>0\right.$ for any $w \in R)=\left(p \in \Gamma ; g^{p}(z)>0\right.$ on $R$ as the function of $\left.z\right)$. Since $g^{w}(p)=0$ $(p \in \Delta)$, we see that

$$
\Gamma_{0} \subset \Gamma-\Delta
$$

Thus, if $p \in \Gamma-\Gamma_{0}$ (resp. $p \in \Gamma_{0}$ ), then $g^{p}(z) \equiv 0$ (resp. $g^{p}(z)>0$ ) on $R$.

Lemma 2.1.1. The function $g^{w}(p)=g^{p}(w)$ is continuous in $(p, w)$ on $R^{*} \times R$ and finitely continuous in $(p, w)$ on $\Gamma \times R$.

Proof. As $g^{w}(z)=g^{z}(w)$ is continuous in $(z, w)$ on $R \times R$, so we have only to show the finite continuity of $g^{w}(p)=g^{p}(w)$ at $\left(p_{0}, w_{0}\right)$ in $\Gamma \times R$. Let $\varepsilon$ be an arbitrary positive number. Since $g^{w_{0}}(p)=g^{p}\left(w_{0}\right)$ is continuous in $p$ on $R^{*}$, we can find a neighborhood $W$ of $p_{0}$ such that $w_{0} \notin W$ and

$$
\left|g^{p}\left(w_{0}\right)-g^{p_{0}}\left(w_{0}\right)\right|<\varepsilon
$$

for any $p$ in $W$. There exists a closure compact open neighborhood $U$ of $w_{0}$ in $R$ such that $R^{*}-\bar{U} \supset \bar{W}$ and $g^{w_{n}}(z)=g^{z}\left(w_{0}\right) \leq N<\infty$ on $R-\bar{U}$. By Harnack's inequality, there exists a neighborhood $V$ of $w_{0}$ such that $\bar{V} \subset U$ and

$$
c^{-1} g^{z}\left(w_{0}\right) \leq g^{z}(w) \leq c g^{z}\left(w_{0}\right)
$$

for any $w$ in $V$ and $z$ in $R-U$, where $c=1+\varepsilon / N$. Hence

$$
\left|g^{z}(w)-g^{z}\left(w_{0}\right)\right|<\varepsilon
$$

for any $w$ in $V$ and $z$ in $R-U$. Since $g^{p}(w)-g^{p}\left(w_{0}\right)$ is continuous in $p$ on $R^{*}$ for any fixed $w$ in $V$ and $R-U$ is dense in $R^{*}-U$, we have

$$
\left|g^{p}(w)-g^{p}\left(w_{0}\right)\right| \leq \varepsilon
$$

for any $w$ in $V$ and $p$ in $R^{*}-U$. Thus for any $(p, w)$ in $W \times V$,

$$
\left|g^{w}(p)-g^{w_{0}}\left(p_{0}\right)\right| \leq\left|g^{p}(w)-g^{p}\left(w_{0}\right)\right|+\left|g^{p}\left(w_{0}\right)-g^{p_{0}}\left(w_{0}\right)\right|<2 \varepsilon,
$$

which shows that $g^{w}(p)=g^{p}(w)$ is continuous in $(p, w)$ at $\left(p_{0}, w_{0}\right)$. Q.E.D. 
LeMmA 2.1.2. Given a point $p$ in $\Gamma_{0}$ (resp. $\Gamma-\Gamma_{0}$ ) and an arbitrary neighborhood $U$ of $p$. Then there exists a decreasing sequence $\left(V_{n}\right)_{n=1}^{\infty}$ of neighborhoods of $p$ such that $\bar{V}_{n} \subset U$ and $\cap_{n=1}^{\infty}\left(V_{n} \cap R\right)=\phi$ and $\lim _{n} \sup _{w \in V_{n} \cap R}\left|g^{p}(z)-g^{w}(z)\right|$ $=0$ uniformly in $z$ on each compact subset of $R$. Hence in particular, $g^{p}(z)$ is positive harmonic (resp. identically zero) on $R$.

Proof. Take a countable dense subset $\left(z_{m}\right)_{m=1}^{\infty}$ of $R$. By induction, we can find sequences $\left(U_{m, n}\right)_{n=1}^{\infty}(m=1,2, \ldots)$ of neighborhoods of $p$ such that

$$
U \supset U_{m, n} \supset U_{m, n+1,} U_{m+1, n}
$$

and $\cap_{n=1}^{\infty}\left(R \cap U_{1, n}\right)=\phi$ and $\lim _{n} \sup _{w_{\in \in U_{m}, n \cap R}}\left|g^{z_{m}}(w)-g^{z_{m}}(p)\right|=0$. This is possible, since $g^{z_{m}}(q)$ is continuous in $q$ at $p$ for each $m=1,2, \ldots$ Set $V_{n}$ $=U_{n, n}$. Then $\lim _{n} \sup _{w \in V_{n} \cap R}\left|g^{z}(w)-g^{z}(p)\right|=0$ for any $z=z_{m}(m=1,2, \ldots)$. Since $\left(z_{m}\right)_{m=1}^{\infty}$ is dense in $R$. by Lemma 2.1.2, $\lim _{n} \sup _{w \in v_{u} \cap R}\left|g^{z}(w)-g^{z}(p)\right|=$ 0 holds for any $z$ in $R$. As $g^{z}(w)=g^{w}(z)$ is harmonic in $z$ on $R$ except $w$, so $\lim _{n} \sup _{w \in V_{n} \cap R}\left|g^{w}(z)-g^{p}(z)\right|=0$ holds for $z$ uniformly on each compact subset of $R$.

Lemma 2.1.3. For any fixed $p$ in $R^{*}, D_{R}\left(g^{p}(z) \cap c\right) \leq 2 \pi c(c>0)$.

Proof. This is clear if $p$ belongs to $R^{*}-\Gamma_{0}$. Hence we have only to treat the case where $p$ belongs to $\Gamma_{0}$. By Lemma 2.1.2, we can find a sequence $\left(w_{n}\right)_{n=1}^{\infty}$ of points in $R$ which do not accumulate in $R$ such that

$$
g^{p}(z)=C-\lim _{n} g^{w_{n}}(z)
$$

on $R$. Then

$$
\lim _{n}\left|\operatorname{grad}\left(g^{w_{n}}(z) \cap c\right)\right|^{2}=\left|\operatorname{grad}\left(g^{p}(z) \cap c\right)\right|^{2}
$$

at each point of $R$ except the set $\left(z \in R ; g^{p}(z)=c\right)$ for each fixed local parameter. Hence by Fatou's lemma and $D\left(g^{w_{n}}(z) \cap c\right)=2 \pi c$, we get

$$
D_{R}\left(g^{p}(z) \cap c\right) \leq \lim \inf _{n} D_{R}\left(g^{w_{n}}(z) \cap c\right)=2 \pi c \quad(c>0) .
$$

2.2. From Lemma 2.1.3, it follows that $g^{p}(z)$ is quasi Dirichlet finite on $R$ for any fixed $p$ in $R^{*}$ and so $g^{p}(z)$ is continuous on $R^{*}$ by Lemma 1.3.1. Hence we can give the following

Definition. The Green kernel $g(p, q)$ on $R^{*}$ is given as the function of $(p, q)$ in $R^{*} \times R^{*}$ by the following double limit:

$$
g(p, q)=\lim _{R \ni z \rightarrow p}\left(\lim _{R \ni w \rightarrow q} g^{w}(z)\right) .
$$


PROPOSITION 1. The Green kernel $g(p, q)$ on $R^{*}$ possesses the following properties:

(g.1) $g(z, w)=g^{w}(z)$ for $(z, w)$ in $R \times R$;

(g.2) $g(z, p)=g(p, z)$ for $z$ in $R$ and $p$ in $R^{*}$;

(g.3) $g(p, q)$ is continuous in $p$ on $R^{*}$ for fixed $q$ in $R^{*}$;

(g.4) $g(z, p)$ is continuous in $(z, p)$ on $R \times R^{*}$ and finitely continuous in $(z, p)$ on $R \times \Gamma$;

(g.5) $g(z, p)$ is harmonic in $z$ on $R-(p)$ for fixed $p$ in $R^{*}$;

(g.6) $g(z, p)>0$ (resp. $\equiv 0)$ on $R$ if $p \in R \cup I_{0}\left(\right.$ resp. $\left.\Gamma-\Gamma_{0}\right)$;

(g.7) $D_{R}(g(z, p) \cap c) \leq 2 \pi c$ for any fixed $p$ in $R^{*}$ and $c>0$;

(g.8) if $q$ is fixed in $\Gamma_{0} \cup R$, then $g(p, q)>0$ for any $p$ in $\Gamma_{0} \cup R$;

(g.9) if $q$ is fixed in $R^{*}$, then $g(p, q)=0$ for $p$ in $\Delta$.

Proof. The properties (g.1)-(g.7) are easy consequences of the definition of $g(p, q)$ and Lemmas 2.1.1, 2,3. To prove (g.8), we have only to treat the case where $q \in \Gamma_{0}$ and $p \in \Gamma_{0}$. We take a normal exhaustion $\left(R_{n}\right)_{n=0}^{\infty}$ of $R$ and we fix a point $w$ in $R_{0}$. Since $g(z, q)\left(q \in \Gamma_{0}\right)$ is a positive harmonic function in $z$ on $R$ by (g.5) and (g.6), we can find a positive number $a$ such that

$$
a g(z, q) \geq g^{w}(z)
$$

on $\partial R_{0}$. As $\operatorname{ag}(z, q) \geq g_{n}^{w}(z)$ on $R_{n}-R_{0}$, so $a g(z, q) \geq g^{w}(z)$ on $R-R_{0}$. By letting $z \rightarrow p \in \Gamma_{0}$, we have

$$
\operatorname{ag}(p, q) \geq g^{w}(p)>0 .
$$

Finally, we prove (g.9). To avoid the trivial case, we assume that $q \in \Gamma_{0}$. By Lemma 2.1.2, we can find a sequence $\left(w_{n}\right)_{n=1}^{\infty}$ of points in $R$ which do not accumulate in $R$ such that $g\left(z, w_{n}\right)$ converges to $g(z, q)$ in $C$-topology on $R$. Hence for any $c>0$,

$$
g(z, q) \cap c=B-\lim _{n} g\left(z, w_{n}\right) \cap c
$$

and since $g(z, q)$ and $g\left(z, w_{n}\right)$ are harmoinc on any compact subset $K$ of $R$ for sufficiently large $n$, we get (cf. p. 73 , Satz 7.4 in [1])

$$
\lim _{n} D_{K}\left(g(z, q) \cap c-g\left(z, w_{n}\right) \cap c\right)=0
$$

and by (g.7),

$$
D\left(g\left(z, w_{n}\right) \cap c\right) \leq 2 \pi c \quad(n=1,2, \ldots) .
$$


As $g\left(z, w_{n}\right) \cap c \in M_{\Delta}(R)$, so by Lemma 1.4.1, $g(z, q) \cap c \in M_{\Delta}(R)$, or $g(p, q) \cap c$ $=0$ in $p$ on $\Delta$. Thus $g(p, q)=0$ for $p$ in $\Delta$.

Q.E.D.

Remark. Notice that we do not claim the general symmetricity of $g$ : $g(p, q)=g(q, p)$. We also do not claim the continuity of $g(p, q)$ in $q$ at $\Gamma_{0}$ for fixed $p$ in $\Gamma_{0}$.

It follow from (g.5) and (g.9) that $g(z, p)\left(p \in \Gamma_{0}\right)$ is a singular positive harmonic function, i.e. the greates harmonic minorant of $g(z, p) \cap c$ is identically zero. In fact, let $u(z)$ be harmonic on $R$ with $0 \leq u(z) \leq g(z, p) \cap c$ on $R$. Then by ( $g .9), 0 \leq \lim \sup _{R \ni z \rightarrow q} u(z) \leq \lim _{R \ni z \rightarrow q} g(z, p) \cap c=g(q, p) \cap c=0$ for any $q$ in $\Delta$. Hence by Lemma $1.2 .4, u(z) \equiv 0$ on $R$.

\section{Quantities concerning Green kernel}

3.1. Let $\Omega$ be an arbitrary non-empty set and $K$ be a mapping of $\Omega \times \Omega$ into $[c, \infty](c>-\infty)$. For each set $X$ in $\Omega$, we set

$$
\left(\begin{array}{l}
n \\
2
\end{array}\right) \tau_{n}(X ; \Omega, K)=\inf _{p_{1}}, \ldots, p_{n} \in X \sum_{i<j}^{1, \ldots, n} K\left(p_{i}, p_{j}\right)
$$

when $X \neq \varnothing$ and $\tau_{n}(X ; \Omega, K)=\infty$ when $X=\varnothing$. Let $p_{1}, \ldots, p_{n+1}$ be arbitrary points in $X$. Then

$$
\sum_{i<j}^{1, \ldots, n+1} K\left(p_{i}, p_{j}\right)=\sum_{i=1}^{k-1} K\left(p_{i}, p_{k}\right)+\sum_{j=k+1}^{n+1} K\left(p_{k}, p_{j}\right)+\sum_{i<j ; i, j \neq k}^{1, \ldots, n+1} K\left(p_{i}, p_{j}\right)
$$

and so

$$
\begin{aligned}
\sum_{i<j}^{1, \ldots, n+1} K\left(p_{i}, p_{j}\right) \geq \sum_{i=1}^{k-1} K\left(p_{i}, p_{k}\right)+\sum_{j=k+1}^{n+1} K\left(p_{k}, p_{j}\right)+\left(\begin{array}{c}
n \\
2
\end{array}\right) \tau_{n}(X ; \Omega, K) \\
(k=1,2, \ldots, n+1) .
\end{aligned}
$$

Summing up these $n+1$ inequality, we get

$$
(n+1) \sum_{i<j}^{1, \ldots, n} K\left(p_{i}, p_{j}\right) \geq 2 \sum_{i<j}^{1, \ldots, n^{n+1}} K\left(p_{i}, p_{j}\right)+(n+1)\left(\begin{array}{l}
n \\
2
\end{array}\right) \tau_{n}(X ; \Omega, K)
$$

or

$$
(n-1) \sum_{i<j}^{1, \ldots, n+1} K\left(p_{i}, p_{j}\right) \geq(n+1)\left(\begin{array}{l}
n \\
2
\end{array}\right) \tau_{n}(X ; \Omega, K) .
$$

Hence we get $(n-1)\left(\begin{array}{c}n+1 \\ 2\end{array}\right) \tau_{n+1}(X ; \Omega, K) \geq(n+1)\left(\begin{array}{c}n \\ 2\end{array}\right) \tau_{n}(X ; \Omega, K)$, or

$$
\tau_{n+1}(X ; \Omega, K) \geq \tau_{n}(X ; \Omega, K) \quad(n=1,2, \ldots) .
$$


Hence we can define

$$
\tau(X ; \Omega, K)=\lim _{n \rightarrow \infty} \tau_{n}(X ; \Omega, K) .
$$

This is called the transfinite diameter of $X$ with respect to $(\Omega, K)$. Similarly we set

$$
n \rho_{n}(X ; \Omega, K)=\sup _{p_{1}, \ldots, p_{n} \in X}\left(\inf _{p \in X} \sum_{i=1}^{n} K\left(p, p_{i}\right)\right)
$$

when $X \neq \varnothing$ aud $\rho_{n}(X ; \Omega, K)=\infty$ when $X=\varnothing$. Let $p_{1}, \ldots, p_{n+m}$ be arbitrary points in $X$. Then

$$
\sum_{i=1}^{n+m} K\left(p, p_{i}\right)=\sum_{i=1}^{n} K\left(p, p_{i}\right)+\sum_{i=n+1}^{m} K\left(p, p_{i}\right)
$$

and so

$$
\inf _{p \in x} \sum_{i=1}^{n} K\left(p, p_{i}\right) \geq \inf _{p \in x} \sum_{i=1}^{n} K\left(p, p_{i}\right)+\inf _{p \in x} \sum_{i=n+1}^{m} K\left(p, p_{i}\right)
$$

Hence

$$
(n+m) \rho_{n+m}(X ; \Omega, K) \geq n \rho_{n}(X ; \Omega, K)+m \rho_{m}(X ; \Omega, K) .
$$

It is well known that for a sequence $\left(a_{n}\right)_{n=1}^{\infty}$ of points in $(-\infty, \infty)$ such that $a_{n+m} \geq a_{n}+a_{m}, \lim _{n} n^{-1} a_{n}$ exists. Hence we can define

$$
\rho(X ; \Omega, K)=\lim _{n \rightarrow \infty} \rho_{n}(X ; \Omega, K) .
$$

This is called the modified Tchebycheff's constant of $X$ with respect to $(\Omega, K)$. Concerning these two quautities $\tau$ and $\rho$, we have

Proposition 2. $\rho(X ; \Omega, K) \geq \tau(X ; \Omega, K)$.

Proof. Let $n>1$ be an arbitrary positive integer. We set $r:=(n-1)^{-1}$ and choose $n$ points $p_{n}, p_{n-1}, \ldots, p_{2}, p_{1}$ in $X$ satisfying

$$
\sum_{j=n-i+1}^{n} K\left(p_{n-i}, p_{j}\right) \leq \inf _{p \in K} \sum_{j=n-i+1}^{n} K\left(p, p_{j}\right)+r \quad(i=1,2, \ldots, n-1) .
$$

We choose these $n$ points inductively as follows. Let $p_{n}$ be an arbitrary point in $X$. Assume that $p_{n}, p_{n-1}, \ldots, p_{n-i+1}(i \leq n-1)$ have been already chosen. Consider

$$
f(p)=\sum_{j=n-i+1}^{n} K\left(p, p_{j}\right)
$$


on $X$. Then, since $\inf _{p \in X} f(p) \geq i c>-\infty$, we can find a point $p_{n-i}$ in $X$ such that $f\left(p_{n-i}\right) \leq \inf _{p \in X} f(p)+r$. This is nothing but (3.1.1) and the induction is completed. By the definition of $\rho_{i}(X ; \Omega, K)$, we get

$$
\inf _{p \in X} \sum_{j=n-i+1}^{n} K\left(p, p_{j}\right) \leq i \rho_{i}(X ; \Omega, K) .
$$

Hence by (3.1.1), we get

$$
\sum_{\jmath=n-i+1}^{n} K\left(p_{n-i}, p_{j}\right) \leq i \rho_{i}(X ; \Omega, K)+r \quad(i=1,2, \ldots, n-1) .
$$

Summing up these $n-1$ inequalities, we get

$$
\sum_{i<j}^{1, \ldots}{ }^{n} K\left(p_{i}, p_{j}\right) \leq \sum_{i=1}^{n-1} i \rho_{i}(X ; \Omega, K)+(n-1) r
$$

and so by the definition of $\tau_{n}(X ; \Omega, K)$, we get

$$
\left(\begin{array}{l}
n \\
2
\end{array}\right) \tau_{n}(X ; \Omega, K) \leq \sum_{i=1}^{n-1} i \rho_{i}(X ; \Omega, K)+1
$$

or

$$
\tau_{n}(X ; \Omega, K) \leq \frac{\sum_{i=1}^{n-1} i \rho_{i}(X ; \Omega, K)}{\left(\begin{array}{c}
n \\
2
\end{array}\right)}+\frac{1}{\left(\begin{array}{c}
n \\
2
\end{array}\right)} .
$$

As $\lim _{i \rightarrow \infty} \rho_{i}(X ; \Omega, K)=\rho(X ; \Omega, K)$, so we can easily see that

$$
\lim _{n \rightarrow \infty} \frac{\sum_{i=1}^{n-1} i \rho_{i}(X ; \Omega, K)}{\left(\begin{array}{l}
n \\
2
\end{array}\right)}=\rho(X ; \Omega, K) .
$$

Hence by making $n \nearrow \infty$ in (3.1.2), we get $\tau(X ; \Omega, K) \leq \rho(X ; \Omega, K)$.

3.2. Let $R$ be a hyperbolic Riemann surface and $R^{*}$ be its Royden's compactification and $g(p, q)$ be the Green kernel on $R^{*}$. For the sake of simplicity, we set, for $X$ in $R^{*}$,

$$
\tau_{n}(X)=\tau_{n}\left(X ; R^{*}, g\right), \quad \tau(X)=\tau\left(X ; R^{*}, g\right)
$$

and similarly

$$
\rho_{n}(X)=\rho_{n}\left(X ; R^{*}, g\right), \quad \rho(X)=\rho\left(X ; R^{*} \cdot g\right)
$$

and we simply say that $\tau(X)$ (resp. $\rho(X))$ the transfinite diameter (resp. modi- 
fied Tchebycheff's constant) of $X$ in $R^{*}$. From the considerations in Section 3.1, we see that

$$
\tau(X)=\lim _{n \rightarrow \infty} \tau_{n}(X), \quad \rho(X)=\lim _{n \rightarrow \infty} \rho_{n}(X)
$$

and

$$
\infty \geq \rho(X) \geq \tau(X) \geq 0 .
$$

3.3. In this section, we state two lemmas concerning Green potentials on hyperbolic Riemann surface. They are well known and contained in the general potential theory on compact metrizable space with positive symmetric kernel (see for example, Ninomiya [11]). But for the sake of completeness, we give proofs for two lemmas following Constantinescu-Cornea's book [1].

Let $\mu$ be a positive regular Borel measure on $R$ and $S_{\mu}$ be the support of $\mu$. The Green potential $g_{\mu}(z)$ is defined by

$$
g_{\mu}(z)=\int g(z, p) d \mu(p) \quad(z \in R)
$$

If $g_{\mu}(z)<\infty$ for a point $z$ in $R$, then $g_{\mu}(z)>0$ and $g_{\mu}(z)$ is harmonic in $R-S_{\mu}$ and $g_{\mu}(z)$ is superharmonic in $R$ (p. 34 in [1]).

A set $A$ in $R$ with $\bar{A} \subset R$ is said to be polar if there exists a positive superharmonic function on $R$ which is infinite on $A$. A property is said to hold quasi everywhere if it holds except a polar set.

The energy $\|\mu\|^{2}$ (resp. mutual energy $\langle\mu, \nu\rangle$ ) of a measure $\mu$ (resp. measures $\mu$ and $\nu$ ) is defined by

$$
\left.\|\mu\|^{2}=\iint g(z, w) d \mu(z) d \mu(w) \text { resp. }\langle\mu, \nu\rangle=\iint g(z, w) d \mu(z) d \nu(w)\right)
$$

For a set $X$ in $R$, we denote by $m_{X}$ the totality of unit positive regular Borel measures $\mu$ on $R$ with $S_{\mu} \subset X$. We put

$$
\gamma(X)=\inf _{\mu \in m_{X}}\|\mu\|^{2}
$$

when $X \neq \emptyset$ and $r(X)=\infty$ when $X=\emptyset$. We say that $1 / \gamma(X)$ is the capacity of $X$ induced by energy integral when $X$ is compact. Then we have

Lemma 3.3.1. Let $F$ be a compact set in $R$ consisting of a finite number of analytic arcs. Then there exists a unique measure $\mu_{0}$ in $m_{F}$ such that $\gamma(F)=$ $\left\|\mu_{0}\right\|^{2}<\infty$ and $g_{\mu_{0}}(z) \leq \gamma(F)$ on $R$.and $g_{\mu}(z)=\gamma(F)$ on $F$. Moreover $g_{\mu_{0}}$ belongs 
to $M_{\Delta}(R)$ and $D_{R}\left(g_{\mu_{0}}(z)\right)=2 \pi r(F)$.

Proof. Let $\left(R_{n}\right)_{n=0}^{\infty}$ be a normal exhaustion of $R$ with $R_{0} \supset F$. By Lemma 1.4.3, there exists a function $w$ in $M(R)$ such that $w=1$ on $F$ and $w=0$ on $\Delta$ and $w$ is harmonic in $R-F$. Hence $w \in M_{\Delta}(R)$ and so we can find $\varphi_{n} \in M_{0}(R)$ such that $w=B D-\lim _{n} \varphi_{n}$ on $R$. If we put $\widetilde{F}$ the sum of arcs $F$ with positive direction and $\operatorname{arcs} F$ with negative direction, then we get

$$
D\left(\varphi_{n}, w\right)=\int_{\tilde{F}} \varphi_{n}^{*} d w
$$

and it implies, by making $n \nearrow \infty$,

$$
D(w)=\int_{\widetilde{F}} * d w=\int_{\partial R_{0}} * d w .
$$

By Frostman's theorem (p. 40 in [1]), there exists a unique positive regular Borel measure $\mu_{1}$ with $S \mu_{1} \subset F$ such that $g_{\mu_{1}}(z) \leq 1$ on $R$ and $g_{\mu_{1}}(z)=1$ quasi everywhere on $F$ and $g_{\mu_{1}}(z) \leq w(z)$ on $R$. Let $s(z)$ be a positive superharmonic function on $R$ with $s(z)=\infty$ on $\left(z \in F ; g_{\mu_{1}}(z)<1\right)$. Then since

$$
\lim \inf _{R \ni z \rightarrow \xi}\left(g_{\mu_{1}}(z)+\varepsilon s(z)-w(z)\right) \geq 0
$$

for any $\xi$ in $F \cup \Delta$ and $\varepsilon>0$, by Lemma 1.2.4, $g_{\mu_{1}}(z)+\varepsilon S(z) \geq w(z)$ on $R$. Hence $g_{\mu_{1}}(z) \geq w(z)$ on $R$ quasi everywhere. As a polar set is measure zero (i.e. the Lebesgue measure of the intersection of polar set with any parameter neighborhood is zero) (p. 31 in [1]), so $g_{\mu_{1}}(z) \geq w(z)$ almost everywhere on $R$ and so $g_{\mu_{1}}(z) \geq w(z)$ everywhere on $R$ (p. 13 in [1]). Hence

$$
w(z)=g_{\mu_{1}}(z)
$$

on, $R$, i.e. $g_{\mu_{1}}(z) \leq 1$ on $R$ and $g_{\mu_{1}}(z)=1$ on $F$. From this, it is clear that $\mu_{1}(F)$ $>0$. Let $\mu \in m_{F}$. Then by energy principle (p. 46 in [1]),

$$
1=\left(\int d_{\mu \mu}\right)^{2}=\left(\int g_{\mu_{1}} d \mu\right)^{2}=\left\langle\mu_{1}, \mu\right\rangle^{2} \leq\left\|\mu_{1}\right\|^{2} \cdot\|\mu\|^{2}
$$

or

$$
\|\mu\|^{2} \geq 1 /\left\|\mu_{1}\right\|^{2} .
$$

On the other hand,

$$
\mu_{1}(F)=\int d \mu_{1}=\int g_{\mu_{1}} d \mu_{1}=\left\|\mu_{1}\right\|^{2}
$$


Hence, if we put $\mu_{0}=\mu_{1} / \mu_{1}(F)$, then $\mu_{0} \in m_{F}$ and

$$
\left\|\mu_{0}\right\|^{2}=\left\|\mu_{1}\right\|^{2} / \mu_{1}(F)^{2}=\left\|\mu_{1}\right\|^{2} /\left\|\mu_{1}\right\|^{1}=1 /\left\|\mu_{1}\right\|^{2} \text {. }
$$

Thus $\|\mu\|^{2} \geq\left\|\mu_{0}\right\|^{2}$ for any $\mu \in m_{F}$ and $\gamma(F)=\left\|\mu_{0}\right\|^{2}=1 / \mu_{1}(F)<\infty$. Hence $g_{\mu_{0}}(z)$ $=r(F) g_{\mu_{1}}(z)$ fulfils the properties that $g_{\mu_{0}}(z) \leq \gamma(F)$ on $R$ and $g_{\mu_{0}}(z)=\gamma(F)$ on $F$. The unicity follows again from Frostman's theorem (p. 40 in [1]).

Notice that $g_{\mu_{0}}=\gamma(F) \cdot w \in M_{\Delta}(R)$. Thus by (3.3.1)

$$
\begin{aligned}
D_{R}\left(g_{\mu_{0}}\right)=\gamma(F)^{2} \cdot \int_{\partial R_{0}} * d w & =r(F) \int_{\partial R_{0}} * d g_{\mu_{0}} \\
& =r(F) \int_{F}\left(\int_{\partial R_{0}} * d g(z, p)\right) d \mu_{0}(p),
\end{aligned}
$$

Since $p$ varies in $R_{0}$ in the last term of the above, it is easy to see that

$$
\int_{\partial R_{0}} * d g(z, p)=2 \pi
$$

Hence by noticing $\mu_{0}(F)=1$, we finally obtain

$$
D_{R}\left(g_{\mu_{0}}\right)=2 \pi \gamma(F) \text {. }
$$

Q.E.D.

Lemma 3.3.2. Let $F$ be as in Lemma 3.3.1. Then $\gamma(F)=\tau(F)=\rho(F) .^{3}$

Proof. For each $n$, we can find points $p_{1}^{(n)}, \ldots, p_{n}^{(n)}$ in $F$ such that

$$
\left(\begin{array}{l}
n \\
2
\end{array}\right) D_{n}(F) \geq \sum_{i<j}^{1, \ldots n^{n}} g\left(p_{i}^{(n)}, p_{j}^{(n)}\right)-1 / n
$$

Let $\mu_{n}$ be defined by $\mu_{n}\left(p_{i}^{(n)}\right)=1 / n(i=1, \ldots, n)$ and $\mu_{n}\left(R-\bigcup_{i=1}^{n}\left(p_{i}^{(n)}\right)\right)=0$. Then $\mu_{n}$ belongs to $m_{F}$. Then there exists a subsequence $\left(\mu_{n_{k}}\right)_{k=1}^{\infty}$ of $\left(\mu_{n}\right)_{n=1}^{\infty}$ such that

$$
\lim _{k \rightarrow \infty} \int f d \mu_{n_{k}}=\int f d \mu
$$

for every finitely continuous function $f$ on $F$ (p. 9 in [1]). Clearly $\mu \in m_{F}$. Let $c>0$. Then by Stone-Weierstrass' theorem, there exists a function

$$
\varphi(z, w)=\sum_{j=1}^{n} a_{j} f_{j}(z) h_{j}(w),
$$

where $a_{j}$ are real numbers and $f_{j}$ and $h_{j}$ are finitely continuous functions on $F$, such that

3) It is well known that this is true for any compact set $F$ in $R$. 


$$
\left|g(z, w) \cap c-\varphi_{n}(z, w)\right|<1 / n
$$

on $F$. Then

$$
\begin{aligned}
& \tau n_{k}(F)+1 / n_{k}>\frac{1}{n_{k}^{2}} \sum_{i=j}^{1,} \varlimsup^{n_{k}} g\left(p_{j}^{\left(n_{k}\right)}, p_{j}^{\left(n_{k}\right)}\right) \geq \iint(g \cap c) d \mu_{n_{k}} d \mu_{n_{k}}-\frac{c}{n_{k}} \\
& \geq \iint \varphi_{n} d \mu_{n_{k}} d \mu_{n_{k}}-\frac{1}{n}-\frac{c}{n_{k}}
\end{aligned}
$$

Since $\iint \varphi_{n} d \mu_{n_{k}} d \mu_{n_{k}}=\sum a_{j} \cdot \int f_{j} d \mu_{n_{k}} \cdot \int h_{j} d \mu_{n_{k}} \rightarrow \sum a_{j} \int f_{j} d \mu \cdot \int h_{j} d \mu=\iint \varphi_{n} d \mu d \mu(k \rightarrow$ $\infty)$, we obtain by making $k \rightarrow \infty$,

$$
\tau(F) \geq \iint \varphi d \mu d \mu-\frac{1}{n} \geq \iint(g \cap c) d \mu d \mu-\frac{2}{n} .
$$

By making $n \nearrow \infty, \tau(F) \geq \iint(g \cap c) d \mu d \mu$. Again by making $c \nearrow_{\infty}, \tau(F) \geq\|\mu\|^{2}$. Thus we get $\tau(F) \geq \gamma(F)$.

By Proposition 2, $\rho(F) \geq \tau(F)$. Hence if we prove $\gamma(F) \geq \rho(F)$, then the proof of Lemma 2 is completed. Let $\mu_{0}$ be as in Lemma 3.3.1 and $p_{1}, \ldots$, $p_{n}$ be arbitrary points in $F$. Then

$$
\gamma(F) \geq \frac{1}{n} \sum_{i=1}^{n} g_{\mu_{0}}\left(p_{i}\right)=\frac{1}{n} \int \sum_{i=1}^{n} g\left(z, p_{i}\right) d \mu_{0}(z) \geq \frac{1}{n} \inf _{p \in F} \sum_{i=1}^{n} g\left(p, p_{i}\right) .
$$

Hence

$$
\gamma(F) \geq \frac{1}{n} \sup _{p_{1}}, \ldots, p_{n} \in F\left(\inf _{p \in F} \sum_{i=1}^{n} g\left(p, p_{i}\right)\right)=\rho_{n}(F)
$$

Thus by making $n \nearrow \infty$, we get $r(F) \geq \rho(F)$,

3.4. Asume that $\Gamma_{0} \neq \varnothing$. Fix a point $z_{0}$ in $R$ and $\left(r_{n}\right)_{n=1}^{\infty}$ be a sequence of positive numbers such that

$$
r_{n}>r_{n+1}, \quad \lim _{n \rightarrow \infty} r_{n}=0
$$

and the level curve $\left(z \in R ; g\left(z, z_{0}\right)=r_{n}\right)$ consists of a countable number of analytic Jordan curves not ending in $R$. Moreover we may assume that the set

$$
U_{n}=\left(z \in R ; g\left(z, z_{0}\right)>r_{n}\right)
$$

is not relatively compact in $R$. We set

$$
\Gamma_{n}=\bar{D}_{n} \cap \Gamma \text {. }
$$


Then $\Gamma_{0}=\bigcup_{n=1}^{\infty} \Gamma$. Let $\left(R_{n}\right)_{n=1}^{\infty}$ be a normal exhaustion of $R$ such that $z_{0} \in R_{1}$. We set

$$
F_{n, m}=\bar{U}_{n} \cap \partial R_{m}
$$

Lemma 3.4.1. The set $U_{n}$ is a subdomain in $R$ and $\bar{U} \cap \Delta=\varnothing$.

Proof. Since $g\left(p, z_{0}\right)=0(p \in \Delta), \bar{U}_{n} \cap \Delta=\emptyset$. Assume, contrary to our assertion, that there exists a component $G$ of $U_{n}$ with $z_{0} \notin G$. Then $g\left(z, z_{0}\right)$ is the bounded harmonic function on $G$ with $g\left(z, z_{0}\right)=r_{n}$ on $\partial G$. Then by Lemma 1.2.4, since $\bar{G} \cap \Delta=\varnothing, g\left(z, z_{0}\right)=r_{n}$ on $G$. This is a contradiction.

Q.E.D.

Lemma 3.4.2. There exists a unique positive harmonic function $w_{n, m}$ on $U_{n+-}-R_{m}$ such that $w_{n, m}=0$ on $\partial U_{n+1}-R_{m}$ and $w_{n, m}=1$ on $F_{n+1, m}$. Moreover, $w_{n, m}$ is continuous on $\bar{U}_{n+1}-\bar{R}_{m}$ and there exists a constant $\sigma_{n}>0$ such that

$$
w_{n, m}(p) \geq \sigma_{n} \text { for any } p \in \Gamma_{n} \quad(m=1,2, \ldots) .
$$

Proof. Let $k>m+1$ and $u_{k}$ be harmonic in $R_{k} \cap U_{n+1}-\bar{R}_{m}$ with boundary value $u_{k}=1$ on $F_{n+1, m}$ and $u_{k}=0$ on $F_{n+1, k} \cup\left(\partial\left(R_{k} \cap U_{n+1}\right)-\bar{R}_{m}\right)$. Since $u_{k} \leq$ $u_{k+p}, w_{n, m}=\lim _{k} u_{k}$ is a positive harmonic function on $U_{n+1}-R_{m}$ with boundary value $w_{n, m}=1$ on $F_{n+1, m}$ and $w_{n, m}=0$ on $\partial U_{n+1}-\bar{R}_{m}$. The unicity of such a $w_{n, m}$ follows from Lemma 1.2.4. We get

$$
D_{R_{k} \cap U_{n+1}-\bar{R}_{m+1}}\left(u_{k}\right)=\int_{R_{m+1} \cap U_{n+1}} u_{k}{ }^{*} d u_{k}
$$

As $\boldsymbol{u}_{k}$ is harmonic on $\partial R_{m+1} \cap \bar{U}_{n+1}$ and converges uniformly to $w_{n, m}$ on $\partial R_{m+1} \cap \bar{U}_{n+1}$, so we get

$$
0 \leq \lim _{k} \int_{\partial R_{m+1} \cap U_{n+1}} \boldsymbol{u}_{k}^{*} d \boldsymbol{u}_{k}=\int_{\partial R_{m+1} \cap U_{n+1}} w_{n, m} * d w_{n, m}<\infty
$$

Hence by Fatou's lemma,

$$
D_{U_{n+1}-} \bar{R}_{m+1}\left(w_{n, m}\right) \leq \lim \inf _{k} D_{R_{k} \cap U_{n+1}-\bar{R}_{m+1}}\left(u_{k}\right)=\int_{\partial k_{m+1} \cap U_{n+1}} w_{n, m} * d w_{n, m}<\infty .
$$

On the other hand, let $v$ be harmonic in $R_{m+1} \cap U_{n+1}-\bar{R}_{m}$ with boundary value $v=w_{n, m}$ on $F_{n+1, m+1}$ and $v=0$ on $\partial\left(R_{m+1} \cap U_{n+1}-\bar{R}_{m}\right)-F_{n+1, m+1}$. Then clearly $D_{R_{m+1} \cap U n+1}-\bar{R}_{m}(v)<\infty$. Let

$$
f= \begin{cases}w_{n, m} & \text { on } R \cap U_{n+1}-\bar{R}_{m+1} \\ v & \text { on } R_{m+1} \cap U_{n+1}-\bar{R}_{m} \\ 0 & \text { on } R-\left(R \cap U_{n+1}-\bar{R}_{m}\right)\end{cases}
$$


Then $f$ is bounded continuous a.c. T function on $R$ and $D_{R}(f)=D_{R_{m+1} \cap U_{n+1}-R_{m}}(v)$ $+D_{U_{n+1}-\bar{B}_{m+1}}\left(w_{n, m}\right)<\infty$. Hence $f \in M(R)$ and so $f$ is continuous on $R^{*}$. In particular, $w_{n, m}$ is continuous on $\bar{U}_{n+1}-R_{m+1}$ and so $\bar{U}_{n+1}-R_{m}$.

Next let $R_{0}=\left(z \in R ; g\left(z, z_{0}\right)>b\right)$ be contained in $R_{1} \cap U_{n+1}$ with its closure. Put

$$
w(z)=\frac{g\left(z, z_{0}\right)-r_{n+1}}{b-r_{n+1}}
$$

Then $w(z)>0$ on $U_{n+1}$ and $w(z)=0$ on $\partial U_{n+1}$ and $w(z)<1$ on $U_{n+1}-\bar{R}_{0}$, Hence $w_{n, m}-w=1-w>0$ on $F_{n+1, m}$ and $w_{n, m}-w=0$ on $\partial U_{n+1}-\bar{R}_{m}$. From this, by Lemma 4 , we get

$$
w_{n, m}(z) \geq w(z) \quad \text { on } \bar{U}_{n+1}-R_{m} .
$$

Let $p$ be in $\Gamma_{n}$. Then

$$
w_{n, m}(p) \geq \frac{g\left(p, z_{0}\right)-r_{n+1}}{b-r_{n+1}} \geq \frac{r_{n}-r_{n+1}}{b-r_{n+1}}=\sigma_{n}>0 \quad(m=1,2, \ldots) .
$$

The unicity of such a $w_{n, m}$ follows from Lemma 1.2.4.

Q.E.D.

LEMMA 3.4.3. $\tau\left(\Gamma_{n}\right) \geq \sigma_{n}^{2} \cdot \tau\left(F_{n+1, m}\right) \quad(m=1,2, \ldots)$.

Proof. Let $k$ be an arbitrary positive integer larger than 4 and $p_{1}, p_{2}, \ldots$, $p_{k}$ be in $\Gamma_{n}$. We choose $k$ points $z_{1}, z_{2}, \ldots, z_{k}$ in $F_{n+1, m}$ inductively as follows. Let

$$
u_{1}(z)=\sum_{i=2}^{k} g\left(z, p_{i}\right)
$$

and $z_{1}$ be in $F_{n+1, m}$ such that

$$
u_{1}\left(z_{1}\right)=\min _{z \in F_{n+1}, m} u_{1}(z) .
$$

Since $u_{1}(z)>0$ on $R, u_{1}(z)-u_{1}\left(z_{1}\right) w_{n, m}(z) \geq 0$ on $\partial\left(U_{n+1}-\bar{R}_{m}\right)$, where $w_{n, m}$ is as in Lemma 3.4.2, and so by Lemma 1.2.4, $u_{1}(z) \geq u_{1}(z) w_{n, m}(z)$ on $U_{n+1}-\bar{R}_{m}$ and so on $\bar{U}_{n+1}-\bar{R}_{m}$. Hence in particular,

$$
u_{1}\left(p_{1}\right) \geq u_{1}\left(z_{1}\right) w_{n, m}\left(p_{1}\right) \geq \sigma_{n} u_{1}\left(z_{1}\right)
$$

and so

$$
\sigma_{n} \sum_{i=2}^{k} g\left(z_{1}, p_{i}\right)+{ }^{2, \ldots}{ }_{i<j}^{k} g\left(p_{i}, p_{j}\right) \leq \sum_{i=2}^{k} g\left(p_{1}, p_{i}\right)+{ }^{2,} \sum_{i<j}{ }^{k} g\left(p_{i}, p_{j}\right)
$$

and hence by putting $a=\sum_{i<j}^{1, \ldots, n} g\left(p_{i}, p_{j}\right)$, we get 


$$
\sigma_{n} \sum_{i=2}^{k} g\left(z_{1}, p_{i}\right)+{ }^{2} \prod_{i<j}^{k} g\left(p_{i}, p_{j}\right) \leq a
$$

Next we choose $z_{2}, \ldots, z_{n-2}$ in $F_{n+1, m}$ satisfying

$$
\begin{aligned}
\sigma_{n}^{2} \sum_{i<j}^{1,} \ldots^{\nu} g\left(z_{i}, z_{j}\right)+\sigma_{n} \sum_{i=1}^{\nu} \sum_{j=\nu+1}^{k} g\left(z_{i}, p_{j}\right)+\sum_{i<j}^{\nu+1, \ldots, k} g\left(p_{i}, p_{j}\right) \leq a \\
(\nu=2,3, \ldots, k-2) .
\end{aligned}
$$

First let

$$
u_{2}(z)=\sum_{j=3}^{k} g\left(z, p_{j}\right)+\sigma_{n} g\left(z_{1}, z\right)
$$

and $z_{2}$ be in $F_{n+1, m}$ such that

$$
u_{1}\left(z_{2}\right)=\min _{z \in F_{n+1}, m} u_{2}(z) .
$$

Similarly as above, we have $u_{2}(p) \geq u_{2}\left(z_{2}\right) w_{n, m}(p) \geq \sigma_{n} u_{2}\left(z_{2}\right)$ for $p$ in $\bar{U}_{n+1}-\bar{R}_{m}$ and so

$$
\sum_{j=3}^{k} g\left(p_{2}, p_{j}\right)+\sigma_{n} g\left(z_{1}, p_{2}\right) \geq \sigma_{n} \sum_{j=3}^{k} g\left(z_{2}, p_{j}\right)+\sigma_{n}^{2} g\left(z_{1}, z_{2}\right) .
$$

From this with (3.4.1), we get

$$
\sigma_{n}^{2} g\left(z_{1}, z_{2}\right)+\sigma_{n} \sum_{i=1}^{2} \sum_{j=2}^{k} g\left(z_{i}, p_{j}\right)+\sum_{i<j}^{3, \ldots, k} g\left(p_{i}, p_{j}\right) \leq a .
$$

This is nothing but (3.4.2) for $\nu=2$. Next assume that $z_{2}, \ldots, z_{\nu}(\nu \leq k-3)$ have been already chosen in $F_{n+1, m}$ satisfying (3.4.2). Let

$$
\boldsymbol{u}_{\nu+1}(z)=\sum_{j=\nu+2}^{k} g\left(z, p_{j}\right)+\sigma_{n} \sum_{i=1}^{\nu} g\left(z_{i}, z\right)
$$

and $z_{\nu+1}$ be in $F_{n+1, m}$ such that

$$
u_{\nu+1}\left(z_{\nu+1}\right)=\min _{z \in \pi_{n+1}, n} u_{\nu+1}(z) .
$$

Similarly as before, we have $u_{\nu+1}(p) \geq u_{\nu+1}\left(z_{\nu+1}\right) w_{n, m}(p) \geq \sigma_{n} u_{\nu+1}\left(z_{\nu+1}\right)$ for $p$ in $\bar{U}_{n+1}-\bar{R}_{m}$ and so

$$
\sum_{j=\nu+2}^{k} g\left(p_{\nu+1}, p_{j}\right)+\sigma_{n} \sum_{i=1}^{\nu} g\left(z_{i}, p_{\nu+1}\right) \geq \sigma_{n} \sum_{j=\nu+2}^{k} g\left(z_{\nu+1}, p_{j}\right)+\sigma_{n}^{2} \sum_{i=1}^{\nu} g\left(z_{i}, z_{\nu+1}\right) .
$$

From this with (3.4.2) for $\nu \leq k-3$, we get

$$
\sigma_{n}^{2} \sum_{i<j}^{1, \ldots+1} g\left(z_{i}, z_{j}\right)+\sigma_{n} \sum_{i=1}^{\nu+1} \sum_{j=\nu+2}^{k} g\left(z_{i}, p_{j}\right)+\sum_{i<j}^{\nu+2, \ldots, k} g\left(p_{i}, p_{j}\right) \leq a .
$$


This is $(3.4 .2)$ for $\nu+1$. Thus we have constructed the system $z_{2}, \ldots, z_{k-2}$. Next let

$$
u_{k-1}(z)=g\left(z, p_{k}\right)+\sigma n \sum_{i=1}^{k-2} g\left(z_{i}, z\right)
$$

and $z_{k-1}$ be in $F_{n+1, m}$ such that

$$
\boldsymbol{u}_{k-1}\left(z_{k-1}\right)=\min _{z \in F_{n+1}, m} u_{k-1}(z) .
$$

Similarly as before, we get $u_{k-1}(p) \geq u_{k-1}\left(z_{k-1}\right) w_{n, m}(p) \geq \sigma_{n} u_{k-1}\left(z_{k-1}\right)$ for $p$ in $\bar{U}_{n+1}-\bar{R}_{m}$ and so

$$
g\left(p_{k-1}, p_{k}\right)+\sigma_{n} \sum_{i=1}^{k-2} g\left(z_{i}, p_{k-1}\right) \geq \sigma_{n} g\left(z_{k-1}, p_{k}\right)+\sigma_{n}^{2} \sum_{i=1}^{k-2} g\left(z_{i}, z_{k-1}\right) .
$$

From this with (3.4.2) for $\nu=k-2$, we get

$$
\sigma_{n}^{2} \sum_{i<j}^{1, \ldots-1} g\left(z_{i}, z_{j}\right)+\sigma n \sum_{i=1}^{k-1} g\left(z_{i}, p_{k}\right) \leq a
$$

Finally let

$$
\boldsymbol{u}_{k}(z)=\sigma_{n} \sum_{i=1}^{k-1} g\left(z_{i}, z\right)
$$

and $z_{k}$ be in $F_{n+1, m}$ such that

$$
u_{k}\left(z_{k}\right)=\min _{z \in F_{n+1, m}} u_{k}(z) .
$$

Similarly as before, we have $u_{k}(p) \geq u_{k}\left(z_{k}\right) w_{n, m}(p) \geq \sigma_{n} u_{k}\left(z_{k}\right)$ for $p$ in $\bar{U}_{n+1}-\bar{R}_{m}$ and so

$$
\sigma_{n} \sum_{i=1}^{k-1} g\left(z_{i}, p_{k}\right) \geq \sigma_{n}^{2} \sum_{i=1}^{k-1} g\left(z_{i}, z_{k}\right)
$$

From this with $(3.4 .3)$, we get

$$
\sigma_{n}^{2} \sum_{i<j}^{1, \ldots}{ }^{k} g\left(z_{i}, z_{j}\right) \leq a
$$

Hence by the definition of $\tau_{k}\left(F_{n+1, m}\right)$, we get

$$
\sigma_{n}^{2}\left(\begin{array}{l}
k \\
2
\end{array}\right) \tau_{k}\left(F_{n+1, m}\right) \leq \sum_{i<j}^{1, \ldots{ }^{k}} g\left(p_{i}, p_{j}\right) .
$$

Since $p_{1}, \ldots, p_{k}$ are arbitrary in $\Gamma_{n}$, so

$$
\sigma_{n}^{2}\left(\begin{array}{l}
k \\
2
\end{array}\right) \tau_{k}\left(F_{n+1, m}\right) \leq\left(\begin{array}{c}
k \\
2
\end{array}\right) \tau_{k}\left(\Gamma_{n}\right)
$$


or $\sigma_{n}^{2} \tau_{k}\left(F_{n+1, m}\right) \leq \tau_{k}\left(\Gamma_{n}\right)$. Hence by making $k \nearrow \infty$, we finally get $\sigma_{n}^{2} \tau\left(F_{n+1, m}\right) \leq \tau\left(\Gamma_{n}\right)$.

LEMMA 3.4.4. $\lim _{m \rightarrow \infty} \gamma\left(F_{n, m}\right)=\infty$.

Proof. Let $\mu_{n, m}$ be as in Lemma 3.3.1 for $F_{n, m}$. Then $D\left(g_{\mu_{n, m}}\right)=2 \pi r\left(F_{n, m}\right)$. Put $u_{m}=g_{\mu_{n, m}} / \gamma\left(F_{n, m}\right)$. Then $u_{m} \in M_{\Delta}(R)$ and $u_{m}=1$ on $F_{n, m}$ and harmonic in $R-F_{n, m}$ and

$$
D\left(u_{m}\right)=2 \pi / \gamma\left(F_{n, m}\right) .
$$

By Lemmas 1.2.1 and 2, there exists $f$ in $M(R)$ such that $f=1$ on $\bar{U}_{n}-R_{m}$ and 0 on $\Delta$. Hence by Lemma 1.4.2, there exists $v_{m} \in M(R)$ such that $v_{m}=1$ on $\bar{U}_{n}-R_{m}$ and $v_{m}=0$ on $\Delta$ and $v_{m}$ is harmonic in $R-\left(\bar{U}_{n}-R_{m}\right)$. Then by applying Lemma 1.4 .2 for $K=F_{n, m}$, we get

$$
D\left(u_{m}\right) \leq D\left(v_{m}\right) \text {. }
$$

Again by applying Lemma 1.4.2 for $K=F_{n, m+p}$ and $v_{m}$ and $v_{m+p}$,

$$
D_{R}\left(v_{m}-v_{m+p}\right)=D_{R}\left(v_{m}\right)-D_{R}\left(v_{m+p}\right) .
$$

Hence $\left(v_{m}\right)_{m=1}^{\infty}$ is $D$-convergent. By Lemma 1.2.4, $v_{m} \geq v_{m+p}$ on $R$ and so $v=$ $\lim _{m} v_{m}$ is a harmonic function on $R$ and so $v=B D-\lim _{m} v_{m}$. Thus $v \in M(R)$ or $v$ is continuous on $R^{*}$ and $0 \leq v \leq v_{m}$ implies $v=0$ on $\Delta$. Hence by Lemma 1.2.4, $v \equiv 0$ on $R$. Thus $B D \cdot \lim v_{m}=0$. From this,

$$
0 \leq \lim \sup _{m} D\left(u_{m}\right) \leq \lim _{m} D\left(v_{m}\right)=0 .
$$

Therefore, we obtain

$$
\lim \inf _{m} \gamma\left(F_{n, m}\right)=\lim _{m} 2 \pi / D\left(u_{m}\right)=\infty,
$$

Proposition 3. $\rho\left(\Gamma_{n}\right)=\infty \quad(n=1,2, \ldots)$.

Proof. By Proposition 2 in Section 3.1 and Lemmas 3.3.2 and 3.4.3, we get the relation

$$
\rho\left(\Gamma_{n}\right) \geq \tau\left(\Gamma_{n}\right) \geq \sigma_{n}^{2} \tau\left(F_{n, m}\right)=\sigma_{n}^{2} \gamma\left(F_{n, m}\right) .
$$

Hence by Lemma 3.4.4, we get, by making $m \nearrow \infty, \mu\left(I_{n}\right)=\infty$.

Q.E.D.

\section{Proofs of Theorems 1 and 2}

4.1. By Proposition 3, $\rho\left(\Gamma_{n}\right)=\infty$. Since $\rho\left(\Gamma_{n}\right)=\lim _{m} \mu_{m}\left(\Gamma_{n}\right)$, we can find an increasing sequence $\left(\boldsymbol{m}_{k}\right)_{k=1}^{\infty}$ of positive integers such that 


$$
\rho_{m_{k}}\left(\Gamma_{n}\right)>2^{k} \quad(k=1,2, \ldots) .
$$

By the definition of $\rho_{m_{k}}\left(\Gamma_{n}\right)$, we can find $m_{k}$ points $p_{k, i}^{(n)}\left(i=1,2, \ldots, m_{k}\right)$ in $\Gamma_{n}$ such that

$$
\inf _{p \in \Gamma_{n}} \sum_{i=1}^{m_{k}} g\left(p, p_{k, i}^{(n)}\right)>2^{k} m_{k}
$$

Then the function

$$
e_{n, k}(p)=2^{-k-1} m_{k}^{-1} \sum_{i=1}^{m_{k}} g\left(p, p_{k, i}^{(n)}\right)
$$

is continuous on $R^{*}$ and harmonic on $R$ and $e_{n, k}(p)>1 / 2$ for any $p$ in $\Gamma_{n}$. Since $g\left(z_{0}, q\right)$ is finitely continuous in $q$ on $\Gamma, g\left(z_{0}, q\right) \leq c_{0}<\infty$ for any $q$ in $\Gamma$. Hence $e_{n, k}\left(z_{0}\right) \leq c_{0} / 2^{k+1}$ and so

$$
e_{n}(z)=\sum_{k=1}^{\infty} e_{n, k}(z)
$$

is a positive harmonic function on $R$. Let $p \in \Gamma_{n}$. As $e_{n}(z)>\sum_{k=1}^{N} e_{n, k}(z)$ on $R$ for any positive integer $N$. Hence

$$
\lim \inf _{R \ni z \rightarrow p} e_{n}(z) \geq \sum_{k=1}^{N} e_{n, k}(p)>\frac{N}{2} .
$$

Next by making $N \nearrow \infty$, we get

$$
\lim _{R \ni z \rightarrow p} e_{n}(z)=\infty \quad\left(p \in \Gamma_{n}\right) .
$$

Now we put, by noticing $e_{n}\left(z_{0}\right) \leq c_{0}(n=1,2, \ldots)$,

$$
e(z)=\sum_{n=1}^{\infty} 2^{-n} e_{n}(z)
$$

on $R$. Then $e(z)$ is a positive harmonic function on $R$. Let $p \in \Gamma_{0}$. Then $p \in$ $I_{n}^{\prime}$ for some $n$. Since $e(z)>2^{-n} e_{n}(z)$ on $R$, we get

$$
\lim \inf _{R \ni z \rightarrow p} e(z) \geq 2^{-n} \lim _{R \ni z \rightarrow p} e_{n}(z)=\infty .
$$

Hence we have

$$
\lim _{R \ni z \rightarrow p} e(z)=\infty \quad\left(p \in \Gamma_{0}\right) .
$$

4.2. we denote by $\varepsilon_{p}\left(p \in R^{*}\right)$ the unit positive regular Borel measure such that $\varepsilon_{p}(p)=1$ and $\varepsilon_{p}\left(R^{*}-(p)\right)=0$. We set

$$
\mu=\sum_{n=1}^{\infty} \sum_{k=1} \sum_{i=1}^{m_{k}} 2^{-n-k-1} m_{k}^{-1} \varepsilon_{p k, i}^{(n)}
$$


Then $\mu$ is a unit positive regular Borel measure on $R^{*}$ with $S_{\mu} \subset \bar{\Gamma}_{0}$ and $\mu\left(R^{*}-\Gamma_{0}\right)=0$ such that

$$
e(z)=\int_{\Gamma_{0}} g(z, q) d \mu(q) .
$$

Clearly, we can write

$$
\mu=\sum_{i=1}^{\infty} t_{i} \varepsilon_{q_{i}}
$$

where $\left(q_{i}\right)_{i=1}^{\infty}$ is the sequence of points in $\Gamma_{0}$ which is a rearangement of $\left(p_{k, i}^{(n)}\right)$, and where $\left(t_{i}\right)_{i=1}^{\infty}$ is the sequence of positive numbers such that $\sum_{i=1}^{\infty} t_{i}=1$ which is given by $t_{i}=2^{-n-k-1} m_{k}^{-1}$ with $q_{i}=p_{k, j}^{(n)}$.

We shall prove that this $\mu$ is required measure in Theorem 2. Clearly $\mu$ satisfies (4) and (5). Notice that

$$
e(z)=\sum_{i=1}^{\infty} t_{i} g\left(z, q_{i}\right)
$$

4.3. Now we show that $D(e(z) \cap c) \leq 2 \pi c(c>0)$ and $e(z)$ is continuous on $R^{*}$ and $e(p)=0$ on $\Delta$ and $e(p)=\infty$ on $\Gamma_{0}$, i.e. $\mu$ satisfies $(6),(7),(8)$ and (9) in Theorem 2.

Let $n$ be an arbitrary but fixed positive integer. We set

$$
x(z)=\sum_{i=1}^{n} t_{i} g\left(z, q_{i}\right)
$$

and

$$
d y(z)=* d x(z)
$$

Then we can use $x+i y$ as local parameter at each point of $R$ except at most a countable number of isolated points in $R$ where $d x(z)=0$. We put

$$
L(\alpha)=\sum_{i=1}^{n} \int_{x=\alpha}\left|* d g\left(z, q_{i}\right)\right|
$$

where $\alpha>0$ and we assume that $d x \neq 0$ on $(z ; x(z)=\alpha)$. As we have

$$
L(\alpha)=\int_{x=\alpha}\left(\sum_{i=1}^{n}\left|\frac{\partial}{\partial x} g\left(z, q_{i}\right)\right|\right) d y,
$$

so by Schwarz's inequality, we obtain

$$
\begin{aligned}
(L(\alpha))^{2} & \leq\left(\int_{x=\alpha}\left(\sum_{i=1}^{n}\left|\frac{\partial}{\partial x} g\left(z, q_{i}\right)\right|^{2}\right) d y\right) \cdot n \int_{x=\alpha} d y \\
& \leq\left(\int_{x=\alpha}\left(\sum_{i=1}^{n}\left|\operatorname{grad} g\left(z, q_{i}\right)\right|^{2} d y\right) \cdot n \int_{x=\alpha} * d x .\right.
\end{aligned}
$$


Notice that except at most a countable number of $\alpha>0, d x \neq 0$ on $(z ; x(z)=\alpha)$. By (g. 3), (g. 7) and (g.9) in Proposition $1, x(z)$ is contiunous on $R^{*}$ and vanishes on $\Delta$ and $x(z) \cap c \in M(R) \quad(c>0)$. Applying (1.4.2) in Lemma 1.4.3 for $K=\left(p \in R^{*} ; x(p) \geq \alpha\right)$ and $(x(z) \cap \alpha) / \alpha$, we get

$$
\alpha^{-2} D_{R}(x \cap \alpha)=D_{R}((x \cap \alpha) / \alpha)=\int_{x=\alpha} * d(x / \alpha)<\infty .
$$

Hence we have

$$
D_{R}(x \cap \alpha)=\alpha \int_{x=\alpha} * d x<\infty .
$$

Thus if $c \leq \alpha \leq c^{\prime}$, where $d x \neq 0$ on $\left(z ; x(z)=c^{\prime}\right)$, then

$$
\int_{x=\alpha} * d x=\alpha^{-1} D_{R}(x \cap \alpha) \leq c^{-1} D_{R}\left(x \cap c^{\prime}\right)<\infty .
$$

Therefore, we get

$$
(L(\alpha))^{2} \leq n c^{-1} D_{R}\left(x \cap c^{\prime}\right) \int_{x=\alpha} \sum_{i=1}^{n}\left|\operatorname{grad} g\left(z, q_{i}\right)\right|^{2} d y,
$$

if $c \leq \alpha \leq c^{\prime}$. Hence

$$
\int_{c \leq x \leq c^{\prime}}(L(x))^{2} d x \leq n c^{-1} D_{R}\left(x \cap c^{\prime}\right) \cdot \sum_{i=1}^{n} D_{R}\left(g\left(z, q_{i}\right) \cap c^{\prime} t_{i}^{-1}\right)<\infty .
$$

Here we used (g.7) in Proposition 1 and the fact that

$$
\begin{aligned}
\int_{c}^{c^{\prime}} d \alpha \int_{x=\alpha}( & \left.\sum_{i=1}^{n}\left|\operatorname{grad} g\left(z, q_{i}\right)\right|^{2}\right) d y \\
& =\sum_{i=1}^{n} \iint_{c \leq x \leq c^{\prime}}\left|\operatorname{grad} g\left(z, q_{i}\right)\right|^{2} d x d y \\
& \leq \sum_{i=1}^{n} \iint_{x \leq c^{\prime}}\left|\operatorname{grad} g\left(z, q_{i}\right)\right|^{2} d x d y .
\end{aligned}
$$

As $g\left(z, q_{i}\right) \leq c^{\prime} t_{i}^{-1}$ on $\left(z \in R ; x(z) \leq c^{\prime}\right)(i=1,2, \ldots, n)$, so

$$
\iint_{x \leq c^{\prime}}\left|\operatorname{grad} g\left(z, q_{i}\right)\right|^{2} d x d y \leq D_{R}\left(g\left(z, q_{i}\right) \cap c^{\prime} t_{i}^{-1}\right) \leq 2 \pi c^{\prime} t_{i}^{-1} .
$$

Hence we get (4.3.2). From (4.3.2), we get $L(\alpha)<\infty$ or

$$
\int_{x=\alpha}\left|d^{*} g\left(z, q_{i}\right)\right|<\infty \quad(i=1,2, \ldots, n)
$$

for almost every $c<\alpha<c^{\prime}$ and so for almost every $\alpha>0$. For the sake of simplicity, we say that $\alpha>0$ is regular for $x(z)$ if $d x \neq 0$ on $(z \in R ; x(z)=\alpha)$ 
and (4.3.3) holds. We notice that the totality of regular positive numbers are dense in the totality of positive numbers.

Now let $c>0$ be regular for $x(z)$. Then by (4.3.1) and (4.3.3), we get

$$
D_{R}(x \cap c)=c \int_{x=c} * d x=c \sum_{i=1}^{n} t_{i} \int_{x=c} * d g\left(z, q_{i}\right) .
$$

Let $\alpha>c t_{i}^{-1}$ and $g\left(z, q_{i}\right) \neq 0$ on $\left(z \in R ; g\left(z, q_{i}\right)=\alpha\right)$. Then the interior of $K^{\prime}=$ $\left(p \in R^{*} ; x(p) \geq c\right)$ contains $K=\left(p \in R^{*} ; g\left(p, q_{i}\right) \geq \alpha\right)$. Since

$$
\int_{x=c}\left|* d g\left(z, q_{i}\right)\right|<\infty
$$

by (4.3.3), we can apply (1.4.2) and (1.4.3) in Lemma 1.4 .3 for $K$ and $K^{\prime}$ and $\left(g\left(z, q_{i}\right) \cap \alpha / \alpha\right)$ and so we get

$$
\begin{aligned}
\int_{x=c} * d\left(\alpha^{-1} g\left(z, q_{i}\right)\right) & =\int_{\partial K^{\prime}} * d\left(\alpha^{-1} g\left(z, q_{i}\right)\right)=\int_{\partial K} * d\left(\alpha^{-1} g\left(z, q_{i}\right)\right) \\
& =D_{R-K}\left(\alpha^{-1} g\left(z, q_{i}\right)\right)=D_{R}\left(\alpha^{-1}\left(g\left(z, q_{i}\right) \cap \alpha\right)\right)
\end{aligned}
$$

or

$$
\int_{x=c} * d g\left(z, q_{i}\right)=\alpha^{-1} D_{R}\left(g\left(z, q_{i}\right) \cap \alpha\right) \leq \alpha^{-1} \cdot 2 \pi \alpha=2 \pi
$$

Hence by (4.3.4), we obtain

$$
D_{R}(x \cap c)=c \sum_{i=1}^{n} t_{i} \int_{x=c} * d g\left(z, q_{i}\right)=2 \pi c \sum_{i=1}^{n} t_{i}<2 \pi c,
$$

i.e.

$$
D_{R}\left(\left(\sum_{i=1}^{n} t_{i} g\left(z, q_{i}\right)\right) \cap c\right)<2 \pi c \quad(n=1,2, \ldots)
$$

As $\left(d\left(\left(\sum_{i=1}^{n} t_{i} g\left(z, q_{i}\right)\right) \cap c\right)\right)_{n=1}^{\infty}$ converges to $d(e(z) \cap c)$ on $R$ except the set $(z ; e(z)=c)$ for each local parameter $z$ on $R$, so by Fatou's lemma,

$$
D_{R}(e(z) \cap c) \leq 2 \pi c .
$$

If $c>0$ is not regular for $x(z)$, we choose regular $c_{n}>0$ for $x(z)$ such that $c_{n} \searrow c$, then $D_{R}(e(z) \cap c) \leq D_{R}\left(e(z) \cap c_{n}\right) \leq 2 \pi c_{n}$. Hence by making $n \nearrow \infty$, we get (4.3.6) for any $c>0$. This is (6) in Theorem 2. From this, by Lemma 1.3.1, $e(z)$ is continuous on $R^{*}$, which is (7) in Theorem 2. From (4.1.1), $e(p)=\infty$ on $\Gamma_{0}$, which is (8). Clearly 


$$
D_{K}\left(\left(\sum_{i=1}^{n} t_{i} g\left(z, q_{i}\right)\right) \cap c-e(z) \cap c\right) \rightarrow 0 \quad(n \rightarrow \infty)
$$

for any compact $K$ in $R$ (cf. p. 78, Satz 7.4 in [1]). Hence by Lemma 1.4.1 with (4.3.6), $e(z) \in M_{\Delta}(R)$, or $e(p)=0$ on 4 , which is (9) in Theorem 2. Thus we proved Theorem 2 completely.

4.4. Theorem 1 follows immeadiately from Theorem 2. In fact, assume that $\left(z_{n}\right)_{n=1}^{\infty} \in \mathscr{F}(R)$. We show that $\lim _{n \rightarrow \infty} g_{\mu}\left(z_{n}\right)=\infty$. If this is not true, then we can find a subsequence $\left(w_{n}\right)_{n=1}^{\infty}$ of $\left(z_{n}\right)_{n=1}^{\infty}$ such that

$$
0 \leq \lim _{n \rightarrow \infty} g_{\mu}\left(w_{n}\right)=b<\infty \text {. }
$$

Clearly $\left(w_{n}\right)_{n=1}^{\infty} \in \mathscr{H}(R)$. Let $p_{0}$ be an accumulation point of $\left(w_{n}\right)_{n=1}^{\infty}$. Then since

$$
\lim \inf _{n \rightarrow \infty} g\left(w_{n}, z_{0}\right)>0,
$$

we conclude that $p_{0} \in \Gamma_{0}$. Let $A=(\lambda)$ be the totality of neighborhoods of $p_{0}$ in $R^{*}$. Then $T=\Lambda \times(1,2,3, \ldots)$ is a directed set if we define that $t=(\lambda, n) \geq$ $t^{\prime}=\left(\lambda^{\prime}, n^{\prime}\right)$ if $\lambda \subset \lambda^{\prime}$ and $n \geq n^{\prime}$. For each $t=(\lambda, n),\left(w_{\nu}\right)_{\nu=1}^{\infty} \cap\left(\lambda-\bar{R}_{n}\right) \neq \varnothing$. We choose a point $w_{t}$ in $\left(w_{\nu}\right)_{\nu=1}^{\infty} \cap\left(\lambda-\bar{R}_{n}\right)$. Then clearly $\lim _{t \in T} w_{t}=p_{0}$. Moreover, let $[t]=n$ if $t=(\lambda, n)$. Then $\lim _{t \in T}[t]=\infty$. Hence by $\lim _{n \rightarrow \infty} g_{\mu}\left(w_{n}\right)=b$, we get $\lim _{t \in T} g_{\mu}\left(w_{t}\right)=b<\infty$. On the other hand, since $g_{\mu}$ is continuous on $R^{*}$ and $g_{\mu}=\infty$ on $I_{0}$, we get

$$
\infty=g_{\mu}\left(p_{0}\right)=\lim _{t \in T} g_{\mu}\left(w_{t}\right)=b<\infty,
$$

which is clearly a contradiction and so $g_{\mu}$ satisfies (1) in Theorem 1 . Since $D\left(g_{\mu} \cap c\right) \leq 2 \pi c, g_{\mu}$ also satisfies $(2)$ in Theorem 1 .

Finally we show that $g_{\mu}$ is singular, which is (3) in Theorem 1 . Let $c>0$ and $h$ be a non-negative harmonic function on $R$ with $h \leq g_{\mu} \cap c$ on $R$. Since $\boldsymbol{g}_{\mu}=0$ on $\Delta$, we get

$$
\lim _{R \ni z \rightarrow q} h(z)=0
$$

for any $q$ in $\Delta$. Hence by Lemma $1.2 .4, h(z) \equiv 0$ on $R$. Thus the greatest harmonic minorant of $g_{\mu} \cap c$ is identically zero and so $g_{\mu}$ is singular.

Thus $u=g_{\mu}$ is the required function in Theorem 1. This completes the proof of Theorem 1 . 


\section{REFERENCES}

[1] C. Constantinescu-A. Cornea: Ideale Ränder Riemannscher Flächen, Springer-Verlag, 1963.

[2] G. C. Evans: Potentials and positively infinite singularites of harmonic functions, Monatsheft für Math. und Phys., 43 (1936), 419-424.

[3] Z. Kuramochi: Mass distributions on the ideal boundaries of abstract Riemann surfaces, I, Osaka Math. J., 8 (1956), 119-137.

[4] Y. Kusunoki-S. Mori: On the harmonic boundary of an open Riemann surface, I, Japanese J. Math., 29 (1960), 52-56.

[5] M. Nakai: On a ring isomorphism induced by quasicoformal mappings, Nagoya Math. J., 14 (1959), 201-221.

[6] - A measure on the harmonic boundary of a Riemann surface, Nagoya Math. J., 17 (1960), 181-218.

[7] - Genus and classification of Riemann surfaces, Osaka Math. J., 14 (1962), 153180.

[ 8 ] - : On Evans potential, Proc. Japan Acad., 38 (1962), 624-629.

[9] —: Evans' harmonic function on Riemann surfaces, Proc. Japan Acad., 39 (1963), 74-78.

[10] - On Evans' solution of the equation $\Delta u=P u$ on Riemann surfaces, Kodai Math. Sem. Rep., 15 (1963), 79-93.

[11] N. Ninomiya: Étude sur la théorie du potential pris par rapport au noyau symétrique, J. Inst. Polytech. Osaka City Univ., 8 (1957), 147-179.

[12] K. Noshiro: Contributions to the theory of singularities of analytic functions, Japanese J. Math., 19 (1948), 299-327.

[13] L. Sario: A linear operator method on arbitrary Riemann surfaces, Trans. Amer. Math. Soc., 72 (1952), 281-295.

[14] H. Selberg: Über die ebenen Punktmengen von der Kapazität Null, Avh. Norske Videnskaps-Acad. Oslo I Math.-Natur, 10 (1937).

\section{Mathematical Institute}

Nagoya University 